Pacific

Journal of

Mathematics

\title{
CONDUCTORS AND NEWFORMS FOR SL(2)
}

JOSHUA M. LANSKY AND A. RAGHURAM 


\title{
CONDUCTORS AND NEWFORMS FOR SL(2)
}

\author{
JOSHUA M. LANSKY AND A. RAGHURAM
}

\begin{abstract}
In this paper we develop a theory of newforms for $\mathrm{SL}_{2}(F)$ where $F$ is a nonarchimedean local field whose residue characteristic is odd. This is analogous to results of Casselman for $\mathrm{GL}_{2}(F)$ and Jacquet, Piatetski-Shapiro, and Shalika for $\operatorname{GL}_{n}(F)$. To a representation $\pi$ of $S_{2}(F)$ we attach an integer $c(\pi)$ that we call the conductor of $\pi$. The conductor of $\pi$ depends only on the $L$-packet $\Pi$ containing $\pi$. It is shown to be equal to the conductor of a minimal representation of $\mathrm{GL}_{2}(F)$ determining the $L$-packet $\Pi$. A newform is a vector in $\pi$ which is essentially fixed by a congruence subgroup of level $c(\pi)$. For $\mathrm{SL}_{2}(F)$ we show that our newforms are always test vectors for some standard Whittaker functionals, and, in doing so, we give various explicit formulae for newforms.
\end{abstract}

\section{Introduction}

To introduce the main theme of this paper we recall the following theorem of Casselman [1973]. Let $F$ be a nonarchimedean local field whose ring of integers is $\mathfrak{O}_{F}$. Let $\mathscr{P}_{F}$ be the maximal ideal of $\mathfrak{O}_{F}$. Let $\psi_{F}$ be a nontrivial additive character of $F$ which is normalized so that the maximal fractional ideal on which it is trivial is $O_{F}$.

Theorem (Casselman). Let $(\pi, V)$ be an irreducible admissible infinite-dimensional representation of $\mathrm{GL}_{2}(F)$. Let $\omega_{\pi}$ denote the central character of $\pi$. Let

$$
\Gamma(m)=\left\{\left(\begin{array}{ll}
a & b \\
c & d
\end{array}\right) \in \mathrm{GL}_{2}\left(\mathscr{O}_{F}\right): c \equiv 0 \quad\left(\bmod \mathscr{P}_{F}^{m}\right)\right\} .
$$

Let

$$
V_{m}=\left\{v \in V: \pi\left(\left(\begin{array}{ll}
a & b \\
c & d
\end{array}\right)\right) v=\omega_{\pi}(d) v, \quad \forall\left(\begin{array}{ll}
a & b \\
c & d
\end{array}\right) \in \Gamma(m)\right\} .
$$

(i) There exists a nonnegative integer $m$ such that $V_{m} \neq(0)$. If $c(\pi)$ denotes the least nonnegative integer $m$ with this property then the epsilon factor

MSC2000: primary 22E50; secondary 22E35, 11S37, 11S40.

Keywords: conductor, newform, SL(2). 
$\epsilon\left(s, \pi, \psi_{F}\right)$ of $\pi$ is up to a constant multiple of the form $q^{-c(\pi) s}$. (Here $q$ is the cardinality of the residue field of $F$.)

(ii) For all $m \geq c(\pi)$ we have $\operatorname{dim} V_{m}=m-c(\pi)+1$.

The assertion $\operatorname{dim} V_{c(\pi)}=1$ is sometimes referred to as multiplicity one for newforms, and the unique vector (up to scalars) in $V_{c(\pi)}$ is called the newform for $\pi$. This is closely related to the classical Atkin-Lehner theory of newforms for holomorphic cusp forms on the upper half-plane [Casselman 1973]. When $c(\pi)=$ $0, \pi$ is a spherical representation and the newform is nothing but the spherical vector.

Newforms play an important role in the theory of automorphic forms. We cite two examples to illustrate this. First, the zeta integral corresponding to the newform is exactly the local $L$-factor associated to $\pi$ (see [Jacquet et al. 1981] for instance). In addition, newforms frequently play the role of test vectors for interesting linear forms associated to $\pi$. For example, the newform is a test vector for an appropriate Whittaker linear functional. In showing this, explicit formulae for newforms are quite often needed. For instance, if $\pi$ is a supercuspidal representation which is realized in its Kirillov model then the newform is the characteristic function of the unit group $\mathcal{O}_{F}^{\times}$. This observation is implicit in [Casselman 1973] and is explicitly stated and proved in [Shimizu 1977]. Since the Whittaker functional on the Kirillov model is given by evaluating functions at $1 \in F^{*}$, we get in particular that the functional is nonzero on the newform. In a related vein, it is shown in [Gross and Prasad 1991] that test vectors for trilinear forms for representations of $\mathrm{GL}_{2}(F)$ are often built from newforms. (See also [Schmidt 2002], where many of these results are documented.)

As far as we know, the only other $p$-adic groups for which there is a theory of newforms are $\mathrm{GL}_{n}(F)$ [Jacquet et al. 1981]; $\mathrm{GL}_{2}(D)$ for a $p$-adic division algebra $D$, [Prasad and Raghuram 2000]; and $\mathrm{GSp}_{4}(F)$ (unpublished work of Brooks Roberts and Ralf Schmidt). In this paper, we propose a theory of newforms and conductors for $\mathrm{SL}_{2}(F)$.

Let $G=\mathrm{SL}_{2}(F)$ where $F$ is a nonarchimedean local field with odd residue characteristic. Crucial to our study of newforms are certain filtrations of maximal compact subgroups of $G$. Let $K=K_{0}=\mathrm{SL}_{2}\left(O_{F}\right)$. Let $K^{\prime}=K_{0}^{\prime}=\alpha^{-1} K_{0} \alpha$ where $\alpha=\left(\begin{array}{cc}\varpi_{F} & 0 \\ 0 & 1\end{array}\right)$. Then $K_{0}$ and $K_{0}^{\prime}$ are, up to conjugacy, the two maximal compact subgroups of $\mathrm{SL}_{2}(F)$. We define filtrations of these maximal compact subgroups as follows. For $m$ an integer $\geq 1$, let

$$
K_{m}=\left\{\left(\begin{array}{cc}
a & b \\
c & d
\end{array}\right) \in \mathrm{SL}_{2}\left(\mathscr{O}_{F}\right): c \equiv 0 \quad\left(\bmod \mathscr{P}_{F}^{m}\right)\right\} \quad \text { and } \quad K_{m}^{\prime}=\alpha^{-1} K_{m} \alpha .
$$


Let $(\pi, V)$ be an irreducible admissible infinite-dimensional representation of $G$. Let $\omega_{\pi}$ be the central character of $\pi$, i.e., the character of $\{ \pm 1\}$ such that

$$
\pi\left(\left(\begin{array}{cc}
-1 & 0 \\
0 & -1
\end{array}\right)\right)=\omega_{\pi}(-1) 1_{V} .
$$

Let $\eta$ be any character of $\mathcal{O}_{F}^{\times}$such that $\eta(-1)=\omega_{\pi}(-1)$. Let $c(\eta)$ denote the conductor of $\eta$. For any $m \geq c(\eta), \eta$ gives a character of $K_{m}$ and $K_{m}^{\prime}$ defined by $\eta\left(\left(\begin{array}{ll}a & b \\ c & d\end{array}\right)\right)=\eta(d)$. We define for $m \geq 0$

$$
\pi_{\eta}^{K_{m}}:=\left\{v \in V: \pi\left(\left(\begin{array}{ll}
a & b \\
c & d
\end{array}\right)\right) v=\eta(d) v, \quad \forall\left(\begin{array}{ll}
a & b \\
c & d
\end{array}\right) \in K_{m}\right\} .
$$

Note that $\pi_{\eta}^{K_{m}}=(0)$ if $m<c(\eta)$. The space $\pi_{\eta}^{K_{m}^{\prime}}$ is defined analogously. We define the $\eta$-conductor $c_{\eta}(\pi)$ of $\pi$ as

$$
c_{\eta}(\pi)=\min \left\{m \geq 0: \pi_{\eta}^{K_{m}} \neq(0) \text { or } \pi_{\eta}^{K_{m}^{\prime}} \neq(0)\right\} .
$$

We define the conductor $c(\pi)$ of $\pi$ by $c(\pi)=\min \left\{c_{\eta}(\pi): \eta\right\}$, where $\eta$ runs over characters of $F^{*}$ such that $\eta(-1)=\omega_{\pi}(-1)$.

We deal with the following basic issues in this paper.

(i) Given an irreducible representation $\pi$, we determine its conductor $c(\pi)$. A very easy consequence (almost built into the definition) is that the conductor depends only on the $L$-packet containing $\pi$.

(ii) We identify the conductor with some other invariants associated to the representation. For instance, for $\mathrm{SL}_{2}(F)$ we show that the conductor of $\pi$ is same as the conductor of a minimal representation of $\mathrm{GL}_{2}(F)$ determining the $L$-packet containing $\pi$. We also determine an explicit relation between our conductor and the notion of depth due to Moy and Prasad (see Section 3.4).

(iii) We determine the growth of the space $\operatorname{dim} V_{\eta}^{K_{m}}$ as a function of $m$. This question is analogous to (ii) of Casselman's theorem quoted above. Computing such dimensions is of importance in local level raising. See [Mann 2001].

(iv) We address the question of whether there is a multiplicity one result for newforms. It turns out that quite often $\operatorname{dim} V_{\eta}^{K_{c(\pi)}}=1$, but this fails in general (see Section 4). In these exceptional cases the dimension of the space of newforms is two.

(v) We prove appropriate Whittaker functionals are nonzero on spaces of newforms. This is of importance in global issues related to newforms. In the proofs, we often need explicit formulae for newforms in various models for the representations. These formulae are interesting for their own sake. For example, if $\psi$ is a character of $F$ of conductor $\widehat{O}_{F}$ and $(\pi, V)$ is a $\psi$-generic supercuspidal representation of $G$, then the newform can be taken as the characteristic function of $\left(O_{F}^{\times}\right)^{2}$ where $V$ is 
regarded as a subspace of the Kirillov model of a canonical minimal representation of $\mathrm{GL}_{2}(F)$ which determines the $L$-packet containing $\pi$; see [Shimizu 1977].

The paper is structured as follows. We briefly summarize preliminaries on representations and $L$-packets of $\mathrm{GL}_{2}(F)$ and $\mathrm{SL}_{2}(F)$ in Section 2. The main results of the paper are given in Section 3. In this section, after stating the definitions, we take up principal series constituents and supercuspidal representations in separate subsections. We also state results comparing the conductor with other invariants of representations. In Section 4 we discuss the multiplicity one issue for newforms for $\mathrm{SL}_{2}(F)$.

We now comment briefly on the proofs. A useful preliminary lemma (Lemma 3.1.3) is proved using a variant of an argument of Deligne [1973] based on Kirillov theory for representations of $\mathrm{GL}_{2}(F)$. This lemma bounds the growth of fixed vectors in representations of $\mathrm{SL}_{2}(F)$.

For subquotients of principal series representations and their $L$-packets, most of the proofs use Mackey theory, convenient double coset decompositions, and details regarding restriction of representations from $\mathrm{GL}_{2}$ to $\mathrm{SL}_{2}$. There are a few surprisingly difficult exceptions. In particular, for the $L$-packet corresponding to a quadratic unramified character we use three different realizations of principal series representations for which a general reference is [Gel'fand et al. 1969].

For supercuspidal representations and their $L$-packets, we make extensive use of Kutzko's construction [1978a; 1978b] of supercuspidal representations of $\mathrm{GL}_{2}(F)$, as well as the analysis of their restrictions to $\mathrm{SL}_{2}(F)$ due to Kutzko and Sally [1983]. In showing that certain vectors are newforms, as in the above mentioned $\mathrm{SL}_{2}$ version of Shimizu's result, we use a combination of arguments involving Kutzko's constructions, the formal Mellin transforms as in Jacquet-Langlands, and the local Langlands correspondence for $\mathrm{GL}_{2}$ (see Propositions 3.3.5 and 3.3.9).

We mention some further directions that arise naturally from this work. To begin with, we hope to show that our theory of newforms and conductors bears upon known results about local factors for $\mathrm{SL}_{2}(F)$. In particular, we believe that our conductors are closely related to the analytic conductors appearing in certain epsilon factors. We also believe that an appropriate zeta-integral corresponding to a newform of a representation is equal to a certain local $L$-factor for that representation. As a possible global application we would like to prove using our newforms that representations (or possibly $L$-packets) of $\mathrm{SL}_{2}$ have a nice rationality field, akin to Waldspurger's [1985] result for $\mathrm{GL}_{2}$.

A companion to this article [Lansky and Raghuram 2004] deals with newforms for the quasisplit unramified unitary group $U(1,1)$. It would be of interest to generalize these results to other groups, namely, to $\mathrm{SL}_{n}$ for higher $n$ and for unitary groups in three variables (for instance the quasisplit unramified unitary group $U(2,1))$. 


\section{Preliminaries}

2.1. Notation. In the following, $F$ will be a fixed nonarchimedean local field whose residue characteristic is odd. Let $\mathcal{O}$ denote its ring of integers and let $\mathscr{P}$ be the maximal ideal of $\mathcal{O}$. Let $\varpi$ be a uniformizer for $F$, i.e., $\mathscr{P}=\varpi \mathcal{O}$. Let $k=\mathscr{O} / \mathscr{P}$ be the residue field of $F$. Let $p$ be the characteristic of $k$ and denote by $q$ the cardinality of $k$. Let $\epsilon$ be an element of $0^{*} \backslash 0^{* 2}$. We will denote by $E$ a quadratic extension of $F$ and by $\omega_{E / F}$ the quadratic character of $F^{*}$ associated to $E / F$ by local class field theory. Recall that the kernel of $\omega_{E / F}$ is $N_{E / F}\left(E^{*}\right)$, the norms from $E^{*}$.

If $n$ is a positive integer, let $U^{n}$ denote the $n$th filtration subgroup $1+\mathscr{P}^{n}$ of $\mathcal{O}^{\times}$, and define $U^{0}=\mathbb{O}^{\times}$. Let $\mathfrak{v}$ denote the additive valuation on $F^{*}$ which takes the value 1 on $\varpi$. We let $|\cdot|$ denote the normalized multiplicative valuation given by $|x|=q^{-\mathfrak{v}(x)}$. If $\chi$ is a character of $F^{*}$ we define the conductor $c(\chi)$ to be the smallest nonnegative integer $n$ such that $\chi$ is trivial on $U^{n}$. Let $\psi$ be a nontrivial additive character of $F$ which is assumed to be trivial on $\mathcal{O}$ and nontrivial on $\mathscr{P}^{-1}$. For any $a \in F$ the character $x \mapsto \psi(a x)$ will be denoted as $\psi_{F, a}$ or simply by $\psi_{a}$.

Let $\widetilde{G}$ denote the group $\mathrm{GL}_{2}(F)$. Let $\widetilde{B}=\widetilde{T} N$ be the standard Borel subgroup of upper triangular matrices in $\widetilde{G}$ with Levi subgroup $\widetilde{T}$ and unipotent radical $N$. Let $\widetilde{Z}$ be the center of $\widetilde{G}$. Let $G=\mathrm{SL}_{2}(F)$. Let $B=T N$ be the standard Borel subgroup of upper triangular matrices in $G$ with Levi subgroup $T$ and unipotent radical $N$. Let $w$ be a representative in the normalizer of $T$ for the nontrivial element of the Weyl group of $T$. Set $K=\mathrm{SL}_{2}(\mathcal{O})$ and $\widetilde{K}=\mathrm{GL}_{2}(\mathcal{O})$. Let $I$ and $\widetilde{I}$ respectively be the standard Iwahori subgroups of $G$ and $\widetilde{G}$.

The following filtrations of maximal compact subgroups of $G$ will be important in our study of newforms. Let $K_{-1}=G$ and $K_{0}=K$. Let $K^{\prime}=K_{0}^{\prime}=\alpha^{-1} K_{0} \alpha$ where $\alpha=\left(\begin{array}{cc}\varpi & 0 \\ 0 & 1\end{array}\right)$. Then $K_{0}$ and $K_{0}^{\prime}$ are, up to conjugacy, the two maximal compact subgroups of $G$. For $m$ an integer $\geq 1$, recall that $K_{m}$ and $K_{m}^{\prime}$ stand for certain congruence subgroups as defined in the introduction.

In addition to $\alpha$, we will also make frequent use of the matrices $\beta:=\left(\begin{array}{ll}1 & 0 \\ 0 & \varpi\end{array}\right)$ and $\gamma:=\left(\begin{array}{cc}\epsilon & 0 \\ 0 & 1\end{array}\right)$.

For any subsets $A, B, C, D \subset F$ we let

$$
\left[\begin{array}{ll}
A & B \\
C & D
\end{array}\right]=\left\{\left(\begin{array}{ll}
a & b \\
c & d
\end{array}\right): a \in A, b \in B, c \in C, d \in D\right\} .
$$

We denote $\left[\begin{array}{cc}1 & \mathscr{P}^{j} \\ 0 & 1\end{array}\right]$ by $N\left(\mathscr{P}^{j}\right)$ or simply by $N(j)$. We let $\bar{N}$ denote the lower triangular unipotent subgroup of $G$ and a similar meaning is given to $\bar{N}\left(\mathscr{P}^{j}\right)$ and $\bar{N}(j)$.

If $\mathscr{H}$ is a closed subgroup of a locally compact unimodular group $\mathscr{G}$ and if $(\sigma, W)$ is a smooth representation of $\mathscr{H}$, then we let $\operatorname{Ind}_{\mathscr{H}}^{\mathscr{G}}(\sigma)$ denote the representation of 
$\mathscr{G}$ induced from $\sigma$, i.e., the space of locally constant functions $f: \varphi \rightarrow W$ such that for all $h \in \mathscr{H}$ and $g \in \mathscr{G}$ we have

$$
f(h g)=\Delta_{\mathscr{H}}^{-1 / 2}(h) \sigma(h) f(g),
$$

where $\Delta_{\mathscr{H}}$ is the modulus character of $\mathscr{H}$. The group $\mathscr{G}$ acts on this space of functions via right translation. We let $\operatorname{ind}_{\mathscr{H}}^{\mathscr{G}}(\sigma)$ denote the subrepresentation of $\operatorname{Ind}_{\mathscr{H}}^{\mathscr{G}}(\sigma)$ consisting of those functions in $\operatorname{Ind}_{\mathscr{H}}^{\mathscr{G}}(\sigma)$ whose supports are compact modulo $\mathscr{H}$.

If $\pi$ is any irreducible representation of $\mathscr{G}$ on which the center of $\mathscr{G}$ acts by a character, we will denote this character by $\omega_{\pi}$. The symbol 11 will denote the trivial representation of the group in context.

For real $\zeta$, let $\lceil\zeta\rceil$ denote the least integer greater than or equal to $\zeta$ and $\lfloor\zeta\rfloor=$ $-\lceil-\zeta\rceil$.

2.2. Some results on $\mathbf{G L}_{2}(\boldsymbol{F})$. We briefly recall Kirillov theory for representations of $\mathrm{GL}_{2}(F)$. For details see [Casselman 1973; Jacquet and Langlands 1970; Prasad and Raghuram 2000]. Let $(\pi, V)$ be an irreducible admissible infinitedimensional representation of $\widetilde{G}=\mathrm{GL}_{2}(F)$. The representation space $V$ may be uniquely realized as a certain space of functions $K(\pi)$, where $C_{c}^{\infty}\left(F^{*}\right) \subset K(\pi) \subset$ $C^{\infty}\left(F^{*}\right)$. Moreover, the space $K(\pi)$ consists of locally constant functions on $F^{*}$ which vanish outside compact subsets of $F$ and the action of $\widetilde{B}$ on $K(\pi)$ is given by the formula

$$
\left(\pi\left(\begin{array}{ll}
a & b \\
0 & d
\end{array}\right) f\right)(x)=\omega_{\pi}(d) \psi\left(d^{-1} b x\right) f\left(d^{-1} a x\right)
$$

for all $a, d, x \in F^{*}$, for all $b \in F$, and for all $f \in K(\pi)$ (see [Casselman 1973]). This Kirillov model $K(\pi)$ has many nice properties, namely:

(i) For all $n \in N$ and for all $v \in V, \pi(n) \phi_{v}-\phi_{v}$ has compact support in $F^{*}$ where $\phi_{v}$ is the function in $K(\pi)$ associated to the vector $v \in V$.

(ii) The space $K(\pi)$ contains $C_{c}^{\infty}\left(F^{*}\right)$ as a subspace of codimension at most two.

(iii) The $\mathbb{C}$-span of functions in (i) is $C_{c}^{\infty}\left(F^{*}\right)$. Or in other words the Jacquet module of $\pi$, denoted $\pi_{N}$, may be identified as a $\widetilde{T}$ module with $K(\pi) / C_{c}^{\infty}\left(F^{*}\right)$.

(iv) The representation $\pi$ is supercuspidal if and only if $C_{c}^{\infty}\left(F^{*}\right)=K(\pi)$.

It is often of interest to know what the newform looks like in the Kirillov model. If $\pi$ is a supercuspidal representation of $\widetilde{G}$ then the characteristic function of $O^{\times}$ is a newform for $\pi$. (This was observed in [Shimizu 1977].) A similar result is known for supercuspidal representations of $\mathrm{GL}_{2}(D)$ [Prasad and Raghuram 2000, Proposition 5.5]. We prove analogous results for $\mathrm{SL}_{2}(F)$ in this paper. 
2.3. L-packets for $\mathrm{SL}_{2}(F)$. In this section we collect statements about the structure of $L$-packets for $G=\mathrm{SL}_{2}(F)$. See [Labesse and Langlands 1979; Gelbart and Knapp 1982; Kutzko and Sally 1983; Shelstad 1979].

If $\widetilde{\pi}$ is an irreducible admissible representation of $\widetilde{G}$, its restriction $\operatorname{Res}_{\mathrm{SL}_{2}(F)} \tilde{\pi}$ to $G$ is a multiplicity-free finite direct sum of irreducible admissible representations $\pi_{1} \oplus \cdots \oplus \pi_{r}$. On the other hand, if $\pi$ is any irreducible admissible representation of $G$, then there exists an irreducible admissible representation $\tilde{\pi}$ of $\widetilde{G}$ whose restriction to $G$ contains $\pi$. The set $\left\{\pi_{1}, \ldots, \pi_{r}\right\}$ is an $L$-packet of $G$ and $\widetilde{G}$ acts transitively on this set. It is known that the cardinality of an $L$-packet is 1,2 or 4 [Shelstad 1979].

Given such a pair $\tilde{\pi}$ and $\pi$, let $X(\tilde{\pi})=\left\{\chi \in \widehat{F^{*}}: \tilde{\pi} \otimes \chi \simeq \tilde{\pi}\right\}$, where we identify a character $\chi$ of $F^{*}$ with the character $\chi \circ$ det of $\widetilde{G}$. Let $\widetilde{G}(\pi)=\left\{g \in \widetilde{G}: s_{\pi} \simeq \pi\right\}$. The representation ${ }^{g} \pi$ is defined as ${ }^{g} \pi(x)=\pi\left(g x g^{-1}\right)$ for all $x \in G$. Clearly, if $\chi \in X(\tilde{\pi})$ then $\chi$, as a character of $\widetilde{G}$, is trivial on $F^{*} G$. (The center of $\widetilde{G}$ is identified with $F^{*}$.) Also $F^{*} G$ is contained in $\widetilde{G}(\pi)$. In fact, given a character $\chi$ of $F^{*}$ we have $\chi \in X(\widetilde{\pi})$ if and only if $\chi$ is trivial on $\widetilde{G}(\pi)$ [Labesse and Langlands 1979, Lemma 2.8].

\section{Newforms for $\mathrm{SL}_{2}$}

3.1. Definitions and the growth lemma. We now give our definition of the conductor of a representation of $G$. Let $(\pi, V)$ be an admissible representation of $G$ admitting a central character which we denote by $\omega_{\pi}$.

We let $\eta$ be any character of $\mathcal{O}^{\times}$such that $\eta(-1)=\omega_{\pi}(-1)$. Let $c(\eta)$ denote the conductor of $\eta$. For any $m \geq c(\eta), \eta$ gives a character of $K_{m}$ and $K_{m}^{\prime}$ defined by $\eta\left(\left(\begin{array}{ll}a & b \\ c & d\end{array}\right)\right)=\eta(d)$.

For any nonnegative integer $m$, we define

$$
\pi_{\eta}^{K_{m}}:=\left\{v \in V: \pi\left(\left(\begin{array}{ll}
a & b \\
c & d
\end{array}\right)\right) v=\eta(d) v, \quad \forall\left(\begin{array}{ll}
a & b \\
c & d
\end{array}\right) \in K_{m}\right\} .
$$

We note that $\pi_{\eta}^{K_{m}}=(0)$ if $m<c(\eta)$. The spaces $\pi_{\eta}^{K_{m}^{\prime}}$ are defined analogously.

We define the $\eta$-conductor $c_{\eta}(\pi)$ of $\pi$ as

$$
c_{\eta}(\pi):=\min \left\{m \geq 0: \pi_{\eta}^{K_{m}} \neq(0) \text { or } \pi_{\eta}^{K_{m}^{\prime}} \neq(0)\right\} .
$$

We define the conductor $c(\pi)$ of $\pi$ by

$$
c(\pi):=\min \left\{c_{\eta}(\pi): \eta\right\}
$$

where $\eta$ runs over characters of $0^{\times}$such that $\eta(-1)=\omega_{\pi}(-1)$. Suppose $\eta$ satisfies $c_{\eta}(\pi)=c(\pi)$. If

$$
\pi_{\eta}^{K_{c(\pi)}} \neq(0) \quad \text { or } \quad \pi_{\eta}^{K_{c(\pi)}^{\prime}} \neq(0)
$$


we call a nonzero element of these spaces a newform of $\pi$, and $\pi_{\eta}^{K_{c(\pi)}}$ or $\pi_{\eta}^{K_{c(\pi)}^{\prime}}$ itself is then called a space of newforms of $\pi$.

The following growth lemma bounds the growth of the dimension of $\pi^{K_{m}}$ for any irreducible representation $\pi$ of $G$. It uses Kirillov theory for $\mathrm{GL}_{2}(F)$. The proof is modeled on Deligne's proof [1973] of a similar $\mathrm{GL}_{2}$ statement of Casselman [1973]. Similar arguments have also been used in the context of $\mathrm{GL}_{2}(D)$ in [Prasad and Raghuram 2000].

Lemma 3.1.3. Let $(\tilde{\pi}, V)$ be an irreducible admissible representation of $\widetilde{G}$. Let $(\pi, V)$ be the restriction of $\tilde{\pi}$ to $G$. For any character $\eta$ of $\mathcal{O}^{\times}$such that $\eta(-1)=$ $\omega_{\tilde{\pi}}(-1)$, and for all $m \geq \max \{c(\eta), 1\}$, we have $\operatorname{dim}\left(\pi_{\eta}^{K_{m}}\right)-\operatorname{dim}\left(\pi_{\eta}^{K_{m-1}}\right) \leq 2$.

Proof. If $V$ is finite-dimensional, then it is one-dimensional so $\pi$ is trivial and $\operatorname{dim}\left(\pi_{\eta}^{K_{m}}\right)=0,1$ for all $m$ and we are done. We henceforth assume that $V$ is infinite dimensional. We also assume that $(\tilde{\pi}, V)$ is realized in its Kirillov model.

Note that $\beta \pi_{\eta}^{K_{m-1}} \subset \pi_{\eta}^{K_{m}}$ since

$$
\beta K_{m-1} \beta^{-1}=\left[\begin{array}{cc}
\mathscr{O}^{\times} & \mathscr{P}^{-1} \\
\mathscr{P}^{m} & \mathscr{O}^{\times}
\end{array}\right] \cap G \supset K_{m}
$$

It suffices to show that $\operatorname{dim}\left(\pi_{\eta}^{K_{m}} / \beta\left(\pi_{\eta}^{K_{m-1}}\right)\right) \leq 2$ for $m \geq \max \{c(\eta), 1\}$.

Let $f \in \pi_{\eta}^{K_{m}}$. Since $f$ is fixed by $N(\mathcal{O}) \subset K_{m}$, we get that $\operatorname{supp}(f) \subset \mathcal{O}$. (Recall that $V$ is in Kirillov model for $\tilde{\pi}$.) Indeed, if for $x \in F^{*}$ we have $f(x) \neq 0$ then

$$
f(x)=\left(\left(\begin{array}{ll}
1 & a \\
0 & 1
\end{array}\right) f\right)(x)=\psi(a x) f(x)
$$

for all $a \in \mathbb{O}$ which implies that $x \in \mathcal{O}$. (Recall that $\psi$ is normalized to be trivial on $\mathcal{O}$ and nontrivial on $\mathscr{P}^{-1}$.) Since $T(\mathcal{O}) \subset K_{m}$ acts via $\eta$ on $f$, we get for all $y \in F^{*}$ and all $u \in \mathbb{O}^{\times}$

$$
\eta\left(u^{-1}\right) f(y)=\left(\left(\begin{array}{cc}
u & 0 \\
0 & u^{-1}
\end{array}\right) f\right)(y)=\omega_{\widetilde{\pi}}\left(u^{-1}\right) f\left(u^{2} y\right),
$$

which gives $f\left(u^{2} y\right)=\left(\omega_{\tilde{\pi}} \eta^{-1}\right)(u) f(y)$. This implies that on $\mathbb{O}^{\times}, f$ is completely determined by its values on 1 and $\epsilon$.

Now suppose $f \in \pi_{\eta}^{K_{m}}$ is such that $\operatorname{supp}(f) \subset \mathscr{P}$. Then we claim that $f \in$ $\beta\left(\pi_{\eta}^{K_{m-1}}\right)$. For this, we show that $\beta^{-1} f \in \pi_{\eta}^{K_{m-1}}$. Note that $\beta^{-1} f$ is $\eta$-fixed by

$$
\beta^{-1} K_{m} \beta \supset\left[\begin{array}{cc}
\mathscr{O}^{\times} & \mathscr{P} \\
\mathscr{P}^{m-1} & \mathcal{O}^{\times}
\end{array}\right] \cap G
$$


and so it suffices to show that $\beta^{-1} f$ is also fixed by $N(\mathbb{O})$. Thus we need to show that for all $y \in F^{*}$ and all $a \in \mathbb{O}$

$$
\left(\left(\begin{array}{ll}
1 & a \\
0 & 1
\end{array}\right) \beta^{-1} f\right)(y)=\left(\beta^{-1} f\right)(y) .
$$

This reduces to $\psi(a y) f(\varpi y)=f(\varpi y)$, which is true from the assumptions on the support of $f$ and the normalization on $\psi$.

Suppose now that $f_{1}, f_{2}, f_{3} \in \pi_{\eta}^{K_{m}}$. Then there exist constants $a_{1}, a_{2}, a_{3}$ such that $\operatorname{supp}\left(a_{1} f_{1}+a_{2} f_{2}+a_{3} f_{3}\right) \subset \mathscr{P}$. By the arguments above, we obtain $a_{1} f_{1}+$ $a_{2} f_{2}+a_{3} f_{3} \in \beta\left(\pi_{\eta}^{K_{m-1}}\right)$. This implies that $\operatorname{dim}\left(\pi_{\eta}^{K_{m}} / \beta\left(\pi_{\eta}^{K_{m-1}}\right)\right)<3$.

3.2. Principal series representations. Let $\chi$ be a character of $F^{*}$. Then $\chi$ inflates to a character of $B$. Let $\pi(\chi)$ stand for the (unitarily) induced representation $\operatorname{Ind}_{B}^{G}(\chi)$. The representation space of $\pi(\chi)$ consists of locally constant complex valued functions $f$ on $G$ such that for all $a \in F^{*}, b \in F$ and $g \in G$, we have

$$
f\left(\left(\begin{array}{cc}
a & b \\
0 & a^{-1}
\end{array}\right) g\right)=|a| \chi(a) f(g) .
$$

The action of $G$ on such functions is by right translation. It is well known that $\pi(\chi)$ is reducible if and only if $\chi$ is either $|\cdot|^{ \pm}$or a nontrivial quadratic character.

There is an essential difference between the two kinds of reducibilities. In the case $\chi=|\cdot|^{ \pm}, \pi(\chi)$ is the restriction to $G$ of a reducible principal series representation of $\widetilde{G}$. Hence $\pi(\chi)$ will have two representations in its Jordan-Hölder series, namely the trivial representation and the Steinberg representation which we will denote by $\mathrm{St}_{G}$.

If $\chi$ is a nontrivial quadratic character, then $\pi(\chi)$ is the restriction to $G$ of an irreducible principal series representation of $\widetilde{G}$ and breaks up as a direct sum of two irreducible representations, which constitute an $L$-packet of $G$. If $\chi=\omega_{E / F}$ we denote $\pi(\chi)$ by $\pi_{E}$ and let $\pi_{E} \simeq \pi_{E}^{1} \oplus \pi_{E}^{2}$. We denote the $L$-packet by $\xi_{E}=$ $\left\{\pi_{E}^{1}, \pi_{E}^{2}\right\}$.

To begin, we need a lemma on double coset decompositions to analyze the space of fixed vectors in principal series representations.

Lemma 3.2.1. Let $m \geq 1$ and set $x_{i}=\left(\begin{array}{cc}1 & 0 \\ \varpi^{i} & 1\end{array}\right)$ and $y_{i}=\left(\begin{array}{cc}1 & 0 \\ \epsilon \varpi^{i} & 1\end{array}\right)$ for $1 \leq i \leq m-1$. A complete set of representatives for the double coset space $K_{m} \backslash K / B(\mathbb{O})$ is given by $\left\{1, w, x_{i}, y_{i}\right\}_{1 \leq i \leq m-1}$.

Proof. Let $k=\left(\begin{array}{ll}a & b \\ c & d\end{array}\right) \in K$. The coset representatives are determined by considering the cases where $c$ is in $\mathscr{P}^{m}$, where $c$ is in $\mathcal{O}^{\times}$, or for $1 \leq i \leq m$, where $\mathfrak{v}(c)=i$ and $\varpi^{i} c^{-1} d$ is or is not a square. We leave the routine details to the reader.

Let $\chi$ be a character of $F^{*}$. Let $\eta$ be a character of $0^{\times}$such that $\eta(-1)=\chi(-1)$. Let $m \geq c(\eta)$. We note that the space $\pi(\chi)_{\eta}^{K_{m}}$ is isomorphic to $\operatorname{Hom}_{K_{m}}(\eta, \pi(\chi))$. 
In light of the Lemma 3.2.1, standard Mackey theory yields for $m \geq 2$

$$
\begin{aligned}
\pi(\chi)_{\eta}^{K_{m}} & =\operatorname{Hom}_{B(\mathcal{O})}(\eta, \chi) \oplus \operatorname{Hom}_{w^{-1} K_{m} w \cap B(\mathcal{O})}\left({ }^{w} \eta, \chi\right) \\
& \oplus \bigoplus_{i=1}^{m-1} \operatorname{Hom}_{x_{i}^{-1} K_{m} x_{i} \cap B(\mathcal{O})}\left({ }^{x_{i}} \eta, \chi\right) \oplus \bigoplus_{i=1}^{m-1} \operatorname{Hom}_{y_{i}^{-1} K_{m} y_{i} \cap B(\mathcal{O})}\left({ }^{y_{i}} \eta, \chi\right)
\end{aligned}
$$

If $m=1$, only the first two terms appear, while if $m=0$, only the first appears. We use this result extensively in the computations of this section.

As mentioned in the introduction, one of the applications of newforms we have in mind is that they are test vectors for Whittaker functionals. For principal series representations and in fact all their subquotients we consider the following $\psi$ Whittaker functional [Schmidt 2002]. For any function $f$ in a principal series representation $\pi(\chi)$ we define

$$
\Lambda_{\psi} f:=\lim _{r \rightarrow \infty} \int_{\mathscr{P}-r} f\left(\left(\begin{array}{cc}
0 & -1 \\
1 & 0
\end{array}\right)\left(\begin{array}{ll}
1 & x \\
0 & 1
\end{array}\right)\right) \bar{\psi}(x) d x,
$$

where the Haar measure $d x$ is normalized such that $\operatorname{vol}(0)=1$.

Proposition 3.2.4 (Unramified principal series representations). Let $\chi$ be an unramified character of $F^{*}$ and let $\pi=\pi(\chi)$ be the corresponding principal series representation of $G$. Then $c(\pi)=0$, and moreover, $c_{\eta}(\pi)=c(\pi)$ only when $\eta$ is trivial. The dimension of the space of fixed vectors under $K_{m}$ is given by

$$
\operatorname{dim} \pi(\chi)^{K_{m}}= \begin{cases}1 & \text { if } m=0, \\ 2 m & \text { if } m \geq 1 .\end{cases}
$$

Proof. Note that Lemma 3.2.1 and the fact that $G=B K$ implies that

$$
\left|K_{m} \backslash G / B\right|=\left|K_{m} \backslash K / B(\mathcal{O})\right|= \begin{cases}1 & \text { if } m=0, \\ 2 m & \text { if } m \geq 1 .\end{cases}
$$

This, together with (3.2.2) proves the proposition.

Corollary 3.2.5 (Test vectors for unramified principal series representations). For an unramified character $\chi$ of $F^{*}$ such that $\chi \neq|\cdot|^{-1}$, let $f_{\text {new }}$ be any nonzero $K$ fixed vector of the representation $\pi(\chi)$. Then we have $\Lambda_{\psi} f_{\text {new }}=L(1, \chi)^{-1} \neq 0$, where $L(s, \chi)$ is the standard local abelian L-factor associated to $\chi$.

Proof. This is a standard computation in the theory of spherical representations. We merely give a sketch of the details. We can take the newform $f=f_{\text {new }}$ to be given by

$$
f(g)=\chi(a)|a|, \quad g=\left(\begin{array}{ll}
a & * \\
0 & a^{-1}
\end{array}\right) k \in B K=G .
$$


We have

$$
\begin{aligned}
\Lambda_{\psi} f_{\text {new }} & =1+\lim _{r \rightarrow \infty} \int_{\mathscr{P}-r \backslash \mathcal{O}} f\left(\left(\begin{array}{cc}
x^{-1} & -1 \\
0 & x
\end{array}\right)\left(\begin{array}{cc}
1 & 0 \\
x^{-1} & 1
\end{array}\right)\right) \bar{\psi}(x) d x \\
& =1+\sum_{m=1}^{\infty} \int_{\varpi^{-m} \mathscr{O}^{\times}} \chi\left(x^{-1}\right)\left|x^{-1}\right| \bar{\psi}(x) d x \\
& =\left(1-\chi(\varpi) q^{-1}\right)=L(1, \chi)^{-1} \neq 0 .
\end{aligned}
$$

Proposition 3.2.6 (Steinberg representation). Let $\mathrm{St}_{G}$ be the Steinberg representation of $G$. Then $c\left(\mathrm{St}_{G}\right)=1$, and moreover, $c_{\eta}\left(\mathrm{St}_{G}\right)=c\left(\mathrm{St}_{G}\right)$ only when $\eta$ is trivial. The dimension of the space of fixed vectors under $K_{m}$ is given by

$$
\operatorname{dim}\left(\mathrm{St}_{G}^{K_{m}}\right)= \begin{cases}0 & \text { if } m=0 \\ 2 m-1 & \text { if } m \geq 1\end{cases}
$$

Proof. The result follows from Proposition 3.2.4 and (3.2.2).

Corollary 3.2.7 (Test vectors for the Steinberg representation). Let the Steinberg representation $\mathrm{St}_{G}$ be realized as the unique irreducible subrepresentation of $\pi(|\cdot|)$. Then the $\psi$-Whittaker functional $\Lambda_{\psi}$ is nonzero on the space of newforms $\left(\mathrm{St}_{G}\right)_{\text {new }}=\mathrm{St}_{G}^{K_{1}}$.

Proof. We consider the standard intertwining operator $M: \pi(|\cdot|) \rightarrow \pi\left(|\cdot|^{-1}\right)$ given by

$$
(M f)(g)=\int_{F} f\left(\left(\begin{array}{cc}
0 & -1 \\
1 & 0
\end{array}\right)\left(\begin{array}{ll}
1 & x \\
0 & 1
\end{array}\right) g\right) d x
$$

for all $f \in \pi(|\cdot|)$ and for all $g \in G$. The representation space of the Steinberg representation is simply the kernel $V_{M}$ of $M$ [Bump 1997, §4.5].

Note that a function $f \in \pi(|\cdot|)^{K_{1}}$ is determined by its values on the elements $1, w \in G$. Let $f_{\text {new }}$ be an element of $\pi(|\cdot|)^{K_{1}}$ determined by $f_{\text {new }}(1)=q$ and $f_{\text {new }}(w)=-1$. It is easy to see that $M f_{\text {new }}=0$ and so $f_{\text {new }}$ is indeed a newform for the Steinberg representation. A computation very much like that in the proof of Corollary 3.2.5 shows that $\Lambda_{\psi} f_{\text {new }}=-\left(1+q^{-1}\right) \neq 0$. We leave the details to the reader. See also [Schmidt 2002].

Proposition 3.2.8 (Ramified principal series representations). Let $\chi$ be a ramified character of $F^{*}$. Let $\pi=\pi(\chi)$ be the corresponding principal series representation of $G$. Let $c(\chi)$ denote the conductor of $\chi$.

(i) We have $c(\pi)=c(\chi)$ and further $c_{\eta}(\pi)=c(\pi)$ only for those characters $\eta$ such that $\eta=\chi^{ \pm}$on the group of units $0^{\times}$. 
(ii) If $\left.\chi^{2}\right|_{\left(\mathscr{O}^{\times}\right)^{2}} \neq 11$ and $\eta=\left.\chi^{ \pm}\right|_{0^{\times}}$then

$$
\operatorname{dim} \pi(\chi)_{\eta}^{K_{m}}= \begin{cases}0 & \text { if } m<c(\chi), \\ 1 & \text { if } m=c(\chi), \\ 2(m-c(\chi))+1 & \text { if } m>c(\chi) .\end{cases}
$$

(iii) If $\left.\chi^{2}\right|_{\left(\mathbb{O}^{\times}\right)^{2}}=11$ and $\eta=\left.\chi^{ \pm}\right|_{\mathbb{O}^{\times}}$then

$$
\operatorname{dim} \pi(\chi)_{\eta}^{K_{m}}= \begin{cases}0 & \text { if } m=0, \\ 2 m & \text { if } m \geq 1=c(\chi) .\end{cases}
$$

Proof. Let $\eta$ be a character of $\mathbb{O}^{\times}$such that $\eta(-1)=\chi(-1)$. Let $m \geq c(\eta)$. We must determine the dimensions of the Hom-spaces in (3.2.2). The space $\operatorname{Hom}_{B(\mathcal{O})}(\eta, \chi)$ is nonzero if and only if $\eta=\chi^{-1}$ as characters of $0^{\times}$. The space $\operatorname{Hom}_{w^{-1} K_{m} w \cap B(\mathcal{C})}\left({ }^{w} \eta, \chi\right)$ is nonzero if and only if $\eta=\chi$ as characters of $\mathcal{O}^{\times}$. For the summands corresponding to $x_{i}$ we observe that $\operatorname{Hom}_{x_{i}^{-1} K_{m} x_{i} \cap B(\mathcal{O})}\left({ }^{x_{i}} \eta, \chi\right) \neq(0)$ if and only if $\eta=\chi$ on $1+\mathscr{P}^{\min \{i, m-i\}}$ and $m-i \geq c(\eta)$. An identical statement holds for the summands corresponding to $y_{i}$. All the assertions in the proposition follow easily from these observations. We leave the details to the reader.

Corollary 3.2.9 (Test vectors for ramified principal series representations). Let $\chi$ be a ramified character of $F^{*}$. Let $\pi=\pi(\chi)$ be the corresponding principal series representation of $G$. Assume that $\pi$ is irreducible. Let $m=c(\chi) \geq 1$ denote the conductor of $\chi$. The space of newforms $\pi(\chi)_{\text {new }}=\pi(\chi)_{\eta}^{K_{c(\chi)}}$ is one-dimensional where $\eta$ is $\chi$ restricted to $\mathcal{O}^{\times}$, and the Whittaker functional $\Lambda_{\psi}$ is nonzero on this space of newforms.

Proof. From the proof of Proposition 3.2.8 it follows that a newform may be taken as the function $f_{\text {new }}$ that is supported on the double coset $B w K_{m}$ and on this coset it is given by

$$
f\left(\left(\begin{array}{cc}
t & * \\
0 & t^{-1}
\end{array}\right) w\left(\begin{array}{ll}
a & b \\
c & d
\end{array}\right)\right)=\chi(t)|t| \chi(d) .
$$

As in the proof of Corollary 3.2.5 it can be shown that $\Lambda_{\psi}\left(f_{\text {new }}\right)=\chi(-1) \neq 0$.

We now consider the $L$-packets $\xi_{E}=\left\{\pi_{E}^{1}, \pi_{E}^{2}\right\}$ where $E / F$ is a quadratic extension. To begin, we take up the case where $E / F$ is ramified.

Proposition 3.2.10 (Ramified principal series $L$-packets). Let $\xi_{E}=\left\{\pi_{E}^{1}, \pi_{E}^{2}\right\}$ with $E / F$ ramified. We have $c\left(\pi_{E}^{1}\right)=c\left(\pi_{E}^{2}\right)=1$. Further, we have for $\eta=\left.\omega_{E / F}\right|_{O^{x}}$

$$
\operatorname{dim}\left(\pi_{E}^{1}\right)_{\eta}^{K_{m}}=\operatorname{dim}\left(\pi_{E}^{2}\right)_{\eta}^{K_{m}}= \begin{cases}0 & \text { if } m=0 \\ m & \text { if } m \geq 1\end{cases}
$$


Proof. Let $E=F(\sqrt{-\lambda})$ where $\lambda$ is either $\varpi$ or $\epsilon \varpi$. Note that $\omega_{E / F}$ is trivial on $\lambda$. Let $\tilde{\pi}_{E}$ be the principal series representation of $\widetilde{G}$ unitarily induced from the character $\left(\begin{array}{ll}a & b \\ 0 & d\end{array}\right) \mapsto \omega_{E / F}(a)$. Then it is easily seen that $\tilde{\pi}_{E} \otimes \omega_{E / F}=\tilde{\pi}_{E}$. (Note that $\tilde{\pi}_{E}$ restricts to the representation $\pi\left(\omega_{E / F}\right)=\pi_{E}=\pi_{E}^{1} \oplus \pi_{E}^{2}$ of $G$.) From Section 2.3 we get that $\omega_{E / F}$ is trivial on $G\left(\pi_{E}^{1}\right)$. Hence $\left(\begin{array}{cc}\lambda & 0 \\ 0 & 1\end{array}\right) \in G\left(\pi_{E}^{1}\right)$, which implies that $\gamma \notin G\left(\pi_{E}^{1}\right)$ or in other words $\gamma$ conjugates $\pi_{E}^{1}$ into $\pi_{E}^{2}$. This also gives that $\gamma$ conjugates $\left(\pi_{E}^{1}\right)_{\eta}^{K_{m}}$ into $\left(\pi_{E}^{2}\right)_{\eta}^{K_{m}}$ for all $m$ and for all permissible $\eta$. Since $\left(\pi_{E}\right)_{\eta}^{K_{m}}=\left(\pi_{E}^{1}\right)_{\eta}^{K_{m}} \oplus\left(\pi_{E}^{2}\right)_{\eta}^{K_{m}}$ the proof follows from (i) and (iii) of Proposition 3.2.8 for $\eta=\omega_{E / F}$ on the units.

Corollary 3.2.11 (Test vectors for ramified principal series $L$-packets). Let $\xi_{E}=$ $\left\{\pi_{E}^{1}, \pi_{E}^{2}\right\}$ with $E / F$ ramified. Then one and only one of the two representations in the packet is $\psi$-generic, say $\pi_{E}^{1}$ (so $\pi_{E}^{2}$ is $\psi_{\epsilon}$-generic). The Whittaker functional $\Lambda_{\psi}$ is nonzero on the one-dimensional space of newforms $\left(\pi_{E}^{1}\right)_{\text {new }}=\left(\pi_{E}^{1}\right)_{\omega_{E / F}}^{K_{1}}$. Any $\psi_{\epsilon}$-Whittaker functional is nonzero on the one-dimensional space of newforms for $\pi_{E}^{2}$.

Proof. The assertions for $\pi_{E}^{1}$ follow exactly as in the proof of Corollary 3.2.9. Conjugating by $\gamma$ proves the assertions for $\pi_{E}^{2}$.

Proposition 3.2.12 (Unramified principal series $L$-packet). Let $\xi_{E}=\left\{\pi_{E}^{1}, \pi_{E}^{2}\right\}$ with $E / F$ unramified. Then $c\left(\pi_{E}^{1}\right)=c\left(\pi_{E}^{2}\right)=0$ and $\eta$ is trivial as in Proposition 3.2.4. One and only one of the two representations, say $\pi_{E}^{1}$, has a nonzero vector fixed by $K_{0}$. The dimensions of the space of fixed vectors under $K_{m}$ and $K_{m}^{\prime}$ for the two representations are as follows:

(i) For $r \geq 0, \operatorname{dim}\left(\left(\pi_{E}^{1}\right)^{K_{r}}\right)=2\left\lfloor\frac{r}{2}\right\rfloor+1=\operatorname{dim}\left(\left(\pi_{E}^{2}\right)^{K_{r}^{\prime}}\right)$.

(ii) For $r \geq 0, \operatorname{dim}\left(\left(\pi_{E}^{1}\right)^{K_{r}^{\prime}}\right)=\max \left\{2\left\lfloor\frac{r-1}{2}\right\rfloor+1,0\right\}=\operatorname{dim}\left(\left(\pi_{E}^{2}\right)^{K_{r}}\right)$.

Proof. Recall our notation that $\pi\left(\omega_{E / F}\right)=\pi_{E}=\pi_{E}^{1} \oplus \pi_{E}^{2}$. As in the ramified principal series $L$-packets case, we see that $\alpha$ conjugates $\pi_{E}^{1}$ to $\pi_{E}^{2}$. The assumption that it is $\pi_{E}^{1}$ which has a nonzero vector fixed under $K_{0}$ gives all the dimensions when $r=0$. Since $K_{1}^{\prime}$ can be conjugated by an element of $G$ inside $K_{0}$, and $K_{1}$ inside $K_{0}^{\prime}$, it follows from Proposition 3.2.4 that all the spaces $\left(\pi_{E}^{1}\right)^{K_{1}},\left(\pi_{E}^{1}\right)^{K_{1}^{\prime}}$, $\left(\pi_{E}^{2}\right)^{K_{1}}$ and $\left(\pi_{E}^{2}\right)^{K_{1}^{\prime}}$ are one-dimensional.

Assume now that $\pi_{E}$ is realized in the space $\bar{V}$ of locally constant functions $f \in L^{2}(F)$ such that $\omega_{E / F}(x)|x| f(x)$ is constant for sufficiently large $x \in F$. For this realization [Gel'fand et al. 1969, Chapter 2, §3.1], the action of $g=\left(\begin{array}{ll}a & b \\ c & d\end{array}\right) \in G$ on an $f \in L^{2}(F)$ as above is given by

$$
\left(\left(\begin{array}{ll}
a & b \\
c & d
\end{array}\right) f\right)(x)=\omega_{E / F}(b x+d)|b x+d|^{-1} f((a x+c) /(b x+d)) .
$$


In this realization, the spherical vector $f_{0} \in\left(\pi_{E}^{1}\right)^{K_{0}}$ is given by

$$
f_{0}(x)= \begin{cases}1 & \text { if } x \in \mathcal{O}, \\ \omega_{E / F}(x)|x|^{-1} & \text { if } x \notin 0 .\end{cases}
$$

We define two elements $f_{1}$ and $f_{2}$ by

$$
f_{1}(x)=\left(\beta f_{0}\right)(x)= \begin{cases}-q & \text { if } x \in \mathscr{P} \\ \omega_{E / F}(x)|x|^{-1} & \text { if } x \notin \mathscr{P}\end{cases}
$$

and

$$
f_{2}(x)=\left(\left(\begin{array}{cc}
\varpi^{-1} & 0 \\
0 & \varpi
\end{array}\right) f_{0}\right)(x)= \begin{cases}-q & \text { if } x \in \mathscr{P}^{2} \\
-q^{-1} \omega_{E / F}(x)|x|^{-1} & \text { if } x \notin \mathscr{P}^{2}\end{cases}
$$

Note that $f_{1} \in\left(\pi_{E}^{2}\right)^{K_{1}}$ and $f_{2} \in\left(\pi_{E}^{1}\right)^{K_{2}}$. Analogous to $f_{1}$ we define for every $c \in \mathbb{C}$ an element $f_{1}^{c}$ in $\pi_{E}$ given by

$$
f_{1}^{c}(x)= \begin{cases}c & \text { if } x \in \mathscr{P}, \\ \omega_{E / F}(x)|x|^{-1} & \text { if } x \notin \mathscr{P} .\end{cases}
$$

We claim that for every $c$ the element $f_{1}^{c}$ is fixed by $K_{2}$. This can be seen using the Iwahori factorization: $K_{2}=\bar{N}\left(\mathscr{P}^{2}\right) T(\mathscr{O}) N(\mathbb{O})$. Clearly, both $T(\mathcal{O})$ and $\bar{N}\left(\mathscr{P}^{2}\right)$ fix $f_{1}^{c}$. Moreover, $N(\mathbb{O})$ fixes $f_{1}^{c}$ if and only if $w f_{1}^{c}$ is fixed by $\bar{N}(\mathcal{O})$. The observation that

$$
\left(w f_{1}^{c}\right)(x)= \begin{cases}1 & \text { if } x \in \mathcal{O}, \\ c \omega_{E / F}(x)|x|^{-1} & \text { if } x \notin 0\end{cases}
$$

shows that $w f_{1}^{c}$ is indeed fixed by $\bar{N}(\mathcal{O})$. Hence, for every $c, f_{1}^{c}$ is fixed by $K_{2}$. Note that for any fixed $c$ the elements $f_{0}, f_{2}, f_{1}^{c}$ are all linearly independent. Further, as $c$ varies the elements $f_{1}^{c}$ span a two-dimensional space. Therefore, we have shown that $\operatorname{dim} \pi_{E}^{K_{2}}$ is at least 4, and, applying Lemma 3.1.3, we see that the dimension is at most four, hence equals 4 . We now need to determine the dimensions of $\left(\pi_{E}^{1}\right)^{K_{2}}$ and $\left(\pi_{E}^{2}\right)^{K_{2}}$ given that their sum is 4 . For this, we need yet another realization of principal series representations for $G$. We will show that there is some $c$ such that $f_{1}^{c}$ is in $\pi_{E}^{1}$ which will then force $\operatorname{dim}\left(\left(\pi_{E}^{1}\right)^{K_{2}}\right)=3$ and $\operatorname{dim}\left(\left(\pi_{E}^{2}\right)^{K_{2}}\right)=1$.

We refer the reader to [Gel'fand et al. 1969, Chapter 2, §3.2] for this third realization, which is obtained by taking Fourier transforms $\widehat{f}$ of functions $f \in \bar{V}$ with respect to $\psi_{\varpi^{-1}}$. We will let $\widehat{V}$ denote the space of all $\widehat{f}$ as $f$ varies over $\bar{V}$.

The representation space of $\pi_{E}^{1}$ can be recognized in $\widehat{V}$ as the space of functions supported only on the norms $N_{E / F}(E)$, and $\pi_{E}^{2}$ as that of functions supported only on nonnorms (see [Gel'fand et al. 1969, Chapter 2, §3.5]). Since $E / F$ is 
unramified, $N_{E / F}(E)$ is the set of elements of $F^{*}$ with has even valuation together with 0 , and the nonnorms are those elements with odd valuation.

Computing the Fourier transform of the spherical vector $f_{0}$ we get that $\pi_{E}^{1}$ consists of functions supported on nonnorms. We will show now that there is a $c$ such that $f_{1}^{c}$ has support inside nonnorms. Indeed, the formula for the Fourier transform of $f_{1}^{c}$ is

$$
\widehat{f_{1}^{c}}\left(\varpi^{n}\right)= \begin{cases}1+c q^{-1} & \text { if } n \text { is odd, } \\ (c-1) q^{-1} & \text { if } n \text { is even. }\end{cases}
$$

Hence for $c=1$ we get that $f_{1}^{c} \in \pi_{E}^{1}$ and for $c=-q$ we get that $f_{1}^{c} \in \pi_{E}^{2}$. We have now computed the dimensions of fixed vectors under $K_{r}$ and $K_{r}^{\prime}$ for $r=0,1,2$. From this point onwards an induction argument takes over.

If the dimensions are known for all $r \leq 2 m$ then using the fact that $K_{r+1}^{\prime}$ can be conjugated inside $K_{r}$ and $K_{r+1}$ inside $K_{r}^{\prime}$, we get using Lemma 3.1.3 that all the spaces $\left(\pi_{E}^{1}\right)^{K_{2 m+1}},\left(\pi_{E}^{1}\right)^{K_{2 m+1}^{\prime}},\left(\pi_{E}^{2}\right)^{K_{2 m+1}}$ and $\left(\pi_{E}^{2}\right)^{K_{2 m+1}^{\prime}}$ have dimensions equal to $2 m+1$. Using the same lemma it suffices now to show that the dimension of $\left(\pi_{E}^{1}\right)^{K_{2 m+2}}$ is at least $2 m+3$.

Using $\left(\pi_{E}^{1}\right)^{K_{2 m+1}}+\left(\pi_{E}^{1}\right)^{K_{2 m+1}^{\prime}} \subset\left(\pi_{E}^{1}\right)^{K_{2 m+2}}$ and that the subgroup of $G$ generated by $K_{2 m+1}$ and $K_{2 m+1}^{\prime}$ is $K_{2 m}^{\prime}$ (which can be seen by Iwahori factorization) we get

$$
\begin{aligned}
\operatorname{dim}\left(\left(\pi_{E}^{1}\right)^{K_{2 m+2}}\right) & \geq \operatorname{dim}\left(\left(\pi_{E}^{1}\right)^{K_{2 m+1}}\right)+\operatorname{dim}\left(\left(\pi_{E}^{1}\right)^{K_{2 m+1}^{\prime}}\right) \\
& =(2 m+1)+(2 m+1)-\operatorname{dim}\left(\left(\pi_{E}^{1}\right)^{K_{2 m+1}} \cap\left(\pi_{E}^{1}\right)^{K_{2 m+1}^{\prime}}\right) \\
& =4 m+2-\operatorname{dim}\left(\left(\pi_{E}^{1}\right)^{K_{2 m}^{\prime}}\right)=4 m+2-(2 m-1)=2 m+3 .
\end{aligned}
$$

Corollary 3.2.13 (Test vectors for unramified principal series $L$-packet). For $E / F$ unramified let $\xi_{E}=\left\{\pi_{E}^{1}, \pi_{E}^{2}\right\}$. Then one and only one of the two representations in the packet is $\psi$-generic, namely $\pi_{E}^{1}$. Moreover, a $\psi$-Whittaker functional is nonzero on the $K_{0}$-fixed vector in $\pi_{E}^{1}$. The representation $\pi_{E}^{2}$ is not $\psi^{\prime}$-generic for any $\psi^{\prime}$ of conductor $\mathbf{O}$. It is $\psi_{\varpi}$-generic and any $\psi_{\varpi}$-Whittaker functional is nonzero on the $K_{0}^{\prime}$-fixed vectors in $\pi_{E}^{2}$.

Proof. The result follows from multiplicity one for Whittaker models, the fact that $\alpha$ conjugates $\pi_{E}^{1}$ to $\pi_{E}^{2}$, and Corollary 3.2.5.

3.3. Supercuspidal representations. We now consider supercuspidal representations of $G=\mathrm{SL}_{2}(F)$. For this we need some preliminaries on how they are constructed. A direct approach is found in Manderscheid's papers [1984]. We, however, use Kutzko's construction [1978a; 1978b] of supercuspidal representations for $\widetilde{G}$ and then the results of [Kutzko and Sally 1983] to obtain information on the supercuspidal representations ( $L$-packets) for $G$. 
We begin by briefly recalling Kutzko's construction of supercuspidal representations of $\widetilde{G}$ via compact induction from very cuspidal representations of maximal open compact-mod-center subgroups. For $l \geq 1$, let $\widetilde{K}(l)=1+\mathscr{P P}^{l} M_{2 \times 2}(\mathcal{O})$ be the principal congruence subgroup of $\widetilde{K}$ of level $l$. Let $\widetilde{K}(0)=\widetilde{K}$. Let $\widetilde{I}$ be the standard Iwahori subgroup consisting of all elements in $\widetilde{K}$ that are upper triangular modulo $\mathscr{P}$. For $l \geq 1$ let $\widetilde{I}(l)=\left[\begin{array}{cc}1+\mathscr{P}^{l} & \mathscr{P}^{l} \\ \mathscr{P}^{l+1} & 1+\mathscr{P}^{l}\end{array}\right]$, and let $\widetilde{I}(0)=\widetilde{I}$. We will let $\widetilde{H}$ denote either $Z \widetilde{K}$ or $N_{\widetilde{G}} \widetilde{I}$, while $\widetilde{J}$ will denote either $\widetilde{K}$ or $\widetilde{I}$ Here $N_{\widetilde{G}} \widetilde{I}$ is the normalizer in $\widetilde{G}$ of $\widetilde{I}$. In either case we let $\widetilde{J}(l)$ denote the corresponding filtration subgroup.

Definition 3.3.1 (Kutzko). An irreducible (and necessarily finite-dimensional) representation $(\widetilde{\sigma}, W)$ of $\widetilde{H}$ is called a very cuspidal representation of level $l \geq 1$ if $\widetilde{\sigma}$ is trivial on $\widetilde{J}(l)$ and $\widetilde{\sigma}$ does not contain the trivial character of $N\left(\mathscr{P}^{l-1}\right)$.

Remark 3.3.2. One easy consequence of the definition is that, if $\widetilde{\sigma}$ is a very cuspidal representation of $Z \widetilde{K}$ (resp. $\left.N_{\widetilde{G}} \widetilde{I}\right)$ of level $l$ then $\operatorname{Hom}_{N(l-1)}(\mathbb{1 1}, \widetilde{\sigma})=$ $\operatorname{Hom}_{\bar{N}(l-1)}(11, \widetilde{\sigma})=(0)\left(\operatorname{resp} \operatorname{Hom}_{N(l-1)}(\mathbb{1}, \tilde{\sigma})=\operatorname{Hom}_{\bar{N}(l)}(\mathbb{1 1}, \widetilde{\sigma})=(0)\right)$.

We say that an irreducible admissible representation $\tilde{\pi}$ of $\widetilde{G}$ is minimal if for every character $\chi$ of $F^{*}$ we have $c(\tilde{\pi}) \leq c(\tilde{\pi} \otimes \chi)$, for $c(\tilde{\pi})$ as in Casselman's Theorem (page 127).

Theorem 3.3.3 [Kutzko 1978a; 1978b]. Let $\pi$ be a minimal irreducible supercuspidal representation of $\widetilde{G}$. Then $\pi$ is compactly induced from a very cuspidal representation $\widetilde{\sigma}$ of one of the two maximal open compact-mod-center subgroups $\widetilde{H}$ of $\widetilde{G}$. Moreover, $\widetilde{H}$ and the equivalence class of $\sigma$ are uniquely determined by $\pi$. If the conductor of $\pi$ is $2 l$ (resp. $2 l+1$ ), then $\pi$ comes from a very cuspidal representation of $Z \widetilde{K}$ (resp. $N_{\widetilde{G}} \widetilde{I}$ ) of level l.

Following Kutzko we use the terminology that a supercuspidal representation of $\widetilde{G}$ is said to be unramified if it comes via compact induction from a representation of $Z \widetilde{K}$ and is said to be ramified otherwise. The theorem assures us that the ramified ones come via compact induction from representations of $N_{\widetilde{G}} \widetilde{I}$. We now take up both types of supercuspidal representations and briefly review how they break up on restriction to $G$ (see [Kutzko and Sally 1983]).

We begin with the unramified case. Let $\widetilde{\sigma}$ be an irreducible very cuspidal representation of $Z \widetilde{K}$ of level $l(\geq 1)$. Let $\tilde{\pi}$ be the corresponding supercuspidal representation of $\widetilde{G}$. Let $\sigma=\operatorname{Res}_{K}(\widetilde{\sigma})$. Then we have $\operatorname{Res}_{G}(\tilde{\pi})=\operatorname{ind}_{K}^{G}(\sigma) \oplus$ ${ }^{\alpha}\left(\operatorname{ind}_{K}^{G}(\sigma)\right)$ where $\alpha=\left(\begin{array}{cc}\varpi & 0 \\ 0 & 1\end{array}\right)$. If $l \geq 2$, or if $l=1$ and $\sigma$ is irreducible, then $\pi=\pi(\sigma)=\operatorname{ind}_{K}^{G}(\sigma)$ is irreducible, hence so is $\pi^{\prime}={ }^{\alpha} \pi$. We thus have an unramified supercuspidal L-packet $\left\{\pi, \pi^{\prime}\right\}$. If $l=1$ and $\sigma$ is reducible, which happens when $\widetilde{\sigma}$ comes from the unique (up to twists) cuspidal representation of $\mathrm{GL}_{2}\left(\mathbb{F}_{q}\right)$ whose restriction to $\mathrm{SL}_{2}\left(\mathbb{F}_{q}\right)$ is reducible and in this case it breaks up into the direct sum of the two cuspidal representations of $\operatorname{SL}_{2}\left(\mathbb{F}_{q}\right)$ of dimension $(q-1) / 2$. 
Correspondingly, we have $\sigma=\sigma_{1} \oplus \sigma_{2}$, and if we let $\pi_{i}=\operatorname{ind}_{K}^{G}\left(\sigma_{i}\right)$ and $\pi_{i}^{\prime}={ }^{\alpha}\left(\pi_{i}\right)$, then we obtain the unique supercuspidal $L$-packet $\left\{\pi_{1}, \pi_{1}^{\prime}, \pi_{2}, \pi_{2}^{\prime}\right\}$ of $G$ containing 4 elements.

For the ramified case let $\widetilde{\sigma}$ be a very cuspidal representation of $N_{\widetilde{G}} \widetilde{I}$ of level $l(\geq 1)$ and let $\tilde{\pi}$ be the corresponding supercuspidal representation of $\widetilde{G}$. Let $\sigma=\operatorname{Res}_{I}(\tilde{\sigma})$. Then $\sigma=\sigma_{1} \oplus \sigma_{2}$ for two irreducible representations $\sigma_{i}(i=1,2)$ of $I$ and $\gamma$ conjugates one to the other, i.e., $\sigma_{2}=\gamma_{\sigma_{1}}$. Let $\pi_{i}=\operatorname{ind}_{I}^{G}\left(\sigma_{i}\right)$ and so $\pi_{2}=\gamma_{\pi_{1}}$. Then the restriction of $\tilde{\pi}$ to $G$ breaks up into the direct sum of two irreducible supercuspidal representations as $\operatorname{Res}_{G}(\tilde{\pi})=\pi_{1} \oplus \pi_{2}$. We call $\left\{\pi_{1}, \pi_{2}\right\}$ a ramified supercuspidal L-packet of $G$.

Proposition 3.3.4 (Unramified supercuspidal $L$-packets of cardinality two). Consider an unramified supercuspidal L-packet $\left\{\pi, \pi^{\prime}\right\}$ determined by a very cuspidal representation $\widetilde{\sigma}$ of level $l$ of $Z \widetilde{K}$ as above. Then the conductors $c(\pi), c\left(\pi^{\prime}\right)$ are both equal to $2 l$. For any character $\eta$ such that $\eta(-1)=\omega_{\pi}(-1)$ we have

(i) $\pi_{\eta}^{K_{2 l-1}}=\pi_{\eta}^{K_{2 l-1}^{\prime}}=\left(\pi^{\prime}\right)_{\eta}^{K_{2 l-1}}=\left(\pi^{\prime}\right)_{\eta}^{K_{2 l-1}^{\prime}}=(0)$.

(ii) If $c(\eta) \leq l$ and $l$ is odd then for all $m \geq 2 l$

(a) $\operatorname{dim} \pi_{\eta}^{K_{m}^{\prime}}=\operatorname{dim}\left(\pi^{\prime}\right)_{\eta}^{K_{m}}=2\left\lceil\frac{m-2 l+1}{2}\right\rceil$.

(b) $\operatorname{dim} \pi_{\eta}^{K_{m}}=\operatorname{dim}\left(\pi^{\prime}\right) \eta_{m}^{K_{m}^{\prime}}=2\left\lfloor\frac{m-2 l+1}{2}\right\rfloor$.

(iii) If $c(\eta) \leq l$ and $l$ is even then for all $m \geq 2 l$

(a) $\operatorname{dim} \pi_{\eta}^{K_{m}}=\operatorname{dim}\left(\pi^{\prime}\right)_{\eta}^{K_{m}^{\prime}}=2\left\lceil\frac{m-2 l+1}{2}\right\rceil$.

(b) $\operatorname{dim} \pi_{\eta}^{K_{m}^{\prime}}=\operatorname{dim}\left(\pi^{\prime}\right)_{\eta}^{K_{m}}=2\left\lfloor\frac{m-2 l+1}{2}\right\rfloor$.

Proof. The statement about the conductors of $\pi, \pi^{\prime}$ follows immediately from (i), (ii), and (iii). To prove (i) it suffices to prove that $\operatorname{Hom}_{K_{2 l-1}}(\eta, \tilde{\pi})=(0)$ where $\widetilde{\pi}=\operatorname{ind}_{Z \widetilde{K}}^{\widetilde{G}}(\widetilde{\sigma})$ is the unramified supercuspidal representation of $\widetilde{G}$ given by the very cuspidal representation $\widetilde{\sigma}$ of level $m$. Using Frobenius reciprocity and Mackey theory [Kutzko 1977] we have

$$
\begin{aligned}
\operatorname{Hom}_{K_{2 l-1}}(\eta, \tilde{\pi}) & =\operatorname{Hom}_{\widetilde{G}}\left(\operatorname{ind}_{K_{2 l-1}}^{\widetilde{G}}(\eta), \operatorname{Ind}_{Z \widetilde{K}}^{\widetilde{G}}(\widetilde{\sigma})\right) \\
& =\prod_{x \in K_{2 l-1} \backslash \widetilde{G} / Z \widetilde{K}} \operatorname{Hom}_{Z \widetilde{K} \cap x^{-1} K_{2 l-1} x}\left({ }^{x} \eta, \widetilde{\sigma}\right) .
\end{aligned}
$$

For brevity, let $I_{x}=\operatorname{Hom}_{Z \widetilde{K} \cap x^{-1} K_{2 l-1} x}\left({ }^{x} \eta, \widetilde{\sigma}\right)$. For every $x$ we will show that $I_{x}=$ (0).

To this end we need a set of representatives for the double cosets. We leave it to the reader to check that this is given by

$$
\begin{aligned}
K_{2 l-1} \backslash \widetilde{G} / Z \widetilde{K}=\left\{\bar{n}(s) h(u) a_{r}: s \in\right. & \left.\mathscr{P} / \mathscr{P}^{2 l-1}, u \in \mathcal{O}^{\times}, r \geq 0\right\} \\
& \cup\left\{\bar{n}(t) w h(u) a_{r}: t \in \mathbb{O} / \mathscr{P}^{2 l-1}, u \in \mathcal{O}^{\times}, r \geq 0\right\},
\end{aligned}
$$


where $\bar{n}(s)=\left(\begin{array}{ll}1 & 0 \\ s & 1\end{array}\right), h(u)=\left(\begin{array}{ll}u & 0 \\ 0 & 1\end{array}\right)$ and $a_{r}=\alpha^{r}=\left(\begin{array}{cc}\varpi^{r} & 0 \\ 0 & 1\end{array}\right)$.

We begin with the case where $x=\bar{n}(s) h(u) a_{r}$. If $s=0$, then $x=h(u) a_{r}$, which implies that $N(\mathcal{O}) \subset Z \widetilde{K} \cap x^{-1} K_{2 l-1} x$ and so

$$
I_{x} \subset \operatorname{Hom}_{N(\mathcal{C})}\left({ }^{x} \eta, \tilde{\sigma}\right)=\operatorname{Hom}_{N(\mathcal{O})}(\mathbb{1}, \tilde{\sigma})=(0)
$$

since $\widetilde{\sigma}$ is very cuspidal. If $s \neq 0$, then $x=h(u) a_{r} \bar{n}\left(u \varpi^{r} s\right)=h(u) a_{r} k$. Since $\bar{n}\left(u \varpi^{r} s\right) \in \widetilde{K}$ we get $I_{x} \simeq I_{h(u) a_{r}}$, and we are reduced to the case $s=0$. Thus $I_{x}=(0)$.

Now let $x=\bar{n}(t) w h(u) a_{r}$. If $r \geq l$, then $N\left(\mathscr{P}^{l-1}\right) \subset Z \widetilde{K} \cap x^{-1} K_{2 l-1} x$, and so

$$
I_{x} \subset \operatorname{Hom}_{N\left(\Phi^{l-1}\right)}\left({ }^{x} \eta, \widetilde{\sigma}\right)=\operatorname{Hom}_{N\left(\Phi^{l-1}\right)}(11, \widetilde{\sigma})=(0)
$$

by Remark 3.3.2. If $r=0$, then $x \in K$, and we have

$$
I_{x} \simeq \operatorname{Hom}_{K_{2 l-1} \cap x Z \widetilde{K} x^{-1}}\left(\eta,{ }^{x} \widetilde{\sigma}\right) \subset \operatorname{Hom}_{N(\mathcal{O})}(11, \widetilde{\sigma})=(0) .
$$

If $0<r<l$ and $t \in \mathscr{P}^{r}$, then rewrite $x$ as $x=g(u) b_{r} k$ where $g(u)=w h(u) w^{-1}$ and $b_{r}=w a_{r} w^{-1}$ and for some $k \in \widetilde{K}$. Here we finish the argument with

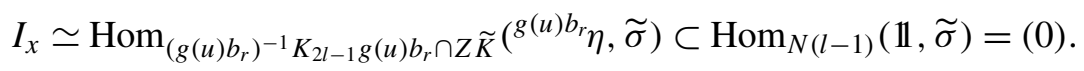

We are finally left with the case where $0<r<l$ and $t \in \mathbb{O}-\mathscr{P}^{r}$. For this, rewrite $x$ as $x=y w$ with $y=\bar{n}(t) b_{r} g(u)$. Since $w \in \widetilde{K}$, as before, we have that $I_{x}=(0)$ if and only if $I_{y}=(0)$. Let $j=j(r, t)=\max \{0, r-2 \mathfrak{v}(t)\}$. Then we get $y^{-1} N\left(\mathscr{P}^{j}\right) y \subset y^{-1} K_{2 l-1} y \cap Z \widetilde{K}$, which gives

$$
I_{y} \subset \operatorname{Hom}_{y^{-1} N\left(\mathscr{P}^{j}\right) y}\left({ }^{y} \eta, \widetilde{\sigma}\right)=\operatorname{Hom}_{y^{-1} N\left(\mathscr{P}^{j}\right) y}(\mathbb{1}, \widetilde{\sigma}) .
$$

We claim that $\operatorname{Hom}_{y^{-1} N\left(\mathscr{P}^{j}\right) y}(\mathbb{1 1}, \widetilde{\sigma})=(0)$. If not there is a nonzero vector $v \in W$ that is fixed by $y^{-1} N\left(\mathscr{P}^{j}\right) y$. Let $v^{\prime}=\widetilde{\sigma}(n(b)) v$ where $b=\varpi^{r} u t^{-1} \in \mathbb{O}$. Then $v^{\prime}$ is fixed by $n(b) y^{-1} N\left(\mathscr{P}^{j}\right) y n(-b)$. Note that

$$
n(b) y^{-1} N\left(\mathscr{P}^{j}\right) y n(-b)= \begin{cases}\bar{N}(\mathcal{O}) & \text { if } r \geq 2 \mathfrak{v}(t) \\ \bar{N}\left(\mathscr{P}^{2 \mathfrak{v}(t)-r}\right) & \text { if } r<2 \mathfrak{v}(t) .\end{cases}
$$

If $r<2 \mathfrak{v}(t)$, then $2 \mathfrak{v}(t)-r \leq l-1$ (by the hypothesis of this case). Hence $v^{\prime}$ is fixed by $\bar{N}\left(\mathscr{P}^{l-1}\right)$, which contradicts that $\widetilde{\sigma}$ is very cuspidal by Remark 3.3.2. This proves (i).

For the proof of (ii) and (iii), we begin by proving that if $l$ is odd, then $\left(\pi^{\prime}\right)^{K_{2 l}}$ has dimension 2, and if $l$ is even, then $\operatorname{dim}\left(\pi^{K_{2 l}}\right)=2$. To this end, let

$$
U=\left\{\left(\begin{array}{cc}
u & 0 \\
0 & u^{-1}
\end{array}\right): u \in \mathbb{O}^{\times}\right\}
$$


Note that $\operatorname{dim} \sigma_{\eta}^{U} \geq 2$. (Recall that $\sigma=\operatorname{Res}_{K} \tilde{\sigma}$. We use the notation $\sigma_{\eta}^{U}$ to stand for the set of all vectors in $\sigma$ on which $U$ acts via $\eta$.) This can be seen as follows. Let $\chi$ be an eigencharacter of $N(O)$ occurring in $\sigma$. Since $\widetilde{\sigma}$ is very cuspidal, $\chi$ has conductor $l$. Let $v_{\chi} \in W$ be a nonzero eigenvector with eigencharacter $\chi$. Let

$$
w_{1}=\sum_{u \in \mathbb{O}^{\times} / \pm(1+\mathscr{P l})} \eta(u) \sigma\left(\left(\begin{array}{cc}
u & 0 \\
0 & u^{-1}
\end{array}\right)\right) v_{\chi}, \quad w_{2}=\tilde{\sigma}(\gamma) w_{1} .
$$

These sums make sense by the hypothesis on $\eta$ and are nonzero since the summands lie in distinct eigenspaces. It is easy to see that $\mathbb{C} w_{1} \oplus \mathbb{C} w_{2} \subset \sigma_{\eta}^{U}$.

For any $w \in \sigma_{\eta}^{U}$ we define two elements $g_{w} \in \operatorname{ind}_{K}^{G}(\sigma)$ and $f_{w} \in \tilde{\pi}$ given by

$$
g_{w}(x)=\left\{\begin{array}{ll}
\sigma(x) w & \text { if } x \in K, \\
0 & \text { if } x \notin K,
\end{array} \quad f_{w}(x)=\tilde{\pi}\left(\left(\begin{array}{cc}
\varpi^{-l} & 0 \\
0 & 1
\end{array}\right)\right) g_{w} .\right.
$$

It is easy to see that $K_{2 l}$ acts via $\eta$ on $f_{w}$, that the map $w \mapsto f_{w}$ is injective, and finally that $f_{w} \in \pi$ if $l$ is even and $f_{w} \in \pi^{\prime}$ if $l$ is odd. Now applying Lemma 3.1.3, we get that if $l$ is even, then $\operatorname{dim} \pi_{\eta}^{K_{2 l}}=2$ and $\left(\pi^{\prime}\right)_{\eta}^{K_{2 l}}=(0)$, whereas if $l$ is odd then $\operatorname{dim}\left(\pi^{\prime}\right)_{\eta}^{K_{2 l}}=2$ and $\pi_{\eta}^{K_{2 l}}=(0)$. (This also shows that $\operatorname{dim} \sigma_{\eta}^{U}=2$.)

Now we use induction to obtain all the dimensions. Let $l$ be even. (We leave the case of odd $l$ to the reader since it is entirely analogous to the even case.) Since for every $m \geq 0$ we have that $K_{m+2} \subset K_{m+1}^{\prime} \subset K_{m}$ up to $G$-conjugacy, it follows from Lemma 3.1.3 that the dimensions of the spaces $\pi_{\eta}^{K_{2 l+1}},\left(\pi^{\prime}\right)_{\eta}^{K_{2 l+1}}, \pi_{\eta}^{K_{2 l+1}^{\prime}}$, and $\left(\pi^{\prime}\right)_{\eta}^{K_{2 l+1}^{\prime}}$ are all equal to 2 . In fact, the same argument shows that if we know the dimensions of $\eta$-fixed vectors under $K_{2 m}$ and $K_{2 m}^{\prime}$, then we would know the dimensions for those under $K_{2 m+1}$ and $K_{2 m+1}^{\prime}$. Let us now suppose that we know $\operatorname{dim} \pi_{\eta}^{K_{i}}$ for $i \leq 2 m+1$. In order to calculate $\operatorname{dim} \pi_{\eta}^{K_{2 m+2}}$, we claim that

$$
\pi_{\eta}^{K_{2 m}} \oplus\left(\begin{array}{cc}
\varpi^{-1} & 0 \\
0 & \varpi
\end{array}\right)^{m-l+1} \pi_{\eta}^{K_{2 l}} \subset \pi_{\eta}^{K_{2 m+2}} .
$$

Clearly, both the summands in the left hand side are contained in the right hand side. We show that the sum is indeed a direct sum. Let $v$ be a vector in the intersection of the two subspaces on the left hand side. Then both

$$
K_{2 l} \text { and }\left(\begin{array}{cc}
\varpi & 0 \\
0 & \varpi^{-1}
\end{array}\right)^{m-l+1} K_{2 m}\left(\begin{array}{cc}
\varpi^{-1} & 0 \\
0 & \varpi
\end{array}\right)^{m-l+1},
$$

and hence $K_{2 l-1}$, act via the character $\eta$ on the vector $\left(\begin{array}{cc}\varpi & 0 \\ 0 & \varpi^{-1}\end{array}\right)^{m-l+1} v$. Hence by (i) we get that $v=0$. This implies that

$$
\operatorname{dim} \pi_{\eta}^{K_{2 m+2}} \geq 2+\operatorname{dim} \pi_{\eta}^{K_{2 m}}=2+\operatorname{dim} \pi_{\eta}^{K^{2 m+1}}
$$


by the induction hypothesis. Lemma 3.1 .3 now says that this is an equality. Conjugating by $\alpha$ gives the dimensions for $\pi^{\prime}$.

Proposition 3.3.5 (Test vectors for unramified supercuspidal $L$-packets of cardinality two). Let $\widetilde{\sigma}$ be a very cuspidal representation of $Z \widetilde{K}$ of level $l$, which determines an unramified supercuspidal L-packet $\left\{\pi, \pi^{\prime}\right\}$ as above. Assume that $\tilde{\pi}=\operatorname{ind}_{Z \widetilde{K}}^{\widetilde{G}}(\widetilde{\sigma})$ is realized in its Kirillov model with respect to $\psi$. Define two elements $\phi_{1}$ and $\phi_{\epsilon}$ in the Kirillov model as follows:

$$
\phi_{1}(x)=\left\{\begin{array}{l}
1 \text { if } x \in\left(\mathbb{O}^{\times}\right)^{2}, \\
0 \text { if } x \notin\left(\mathcal{O}^{\times}\right)^{2},
\end{array} \quad \phi_{\epsilon}(x)=\tilde{\pi}(\gamma) \phi_{1} .\right.
$$

Let $\eta=\omega_{\tilde{\pi}}$. We have

(i) $\mathbb{C} \phi_{1} \oplus \mathbb{C} \phi_{\epsilon}=\tilde{\pi}_{\eta}^{K_{2 l}}$.

(ii) Ifl is even then $\pi_{\eta}^{K_{2 l}}=\tilde{\pi}_{\eta}^{K_{2 l}}$. In addition, $\pi$ is $\psi$-generic and any $\psi$-Whittaker functional is nonzero on $\phi_{1}$ and vanishes on $\phi_{\epsilon}$. Furthermore, $\pi^{\prime}$ is not $\psi^{\prime}$ generic for any character $\psi^{\prime}$ of conductor 0 . It is however $\psi_{\varpi}$-generic and any $\psi_{\varpi}$-Whittaker functional is nonvanishing on $\tilde{\pi}\left(\alpha^{-1}\right) \phi_{1}$ which is a newform for $\pi^{\prime}$.

(iii) If l is odd, then (ii) holds with $\pi$ and $\pi^{\prime}$ interchanged.

Proof. We show that $K_{2 l}$ acts via $\eta$ on $\phi_{1}$. Given this, the rest of the assertions are all quite easy to show using the facts that $\alpha$ conjugates $\pi$ to $\pi^{\prime}, \gamma$ conjugates $K_{2 l}$ to itself, and the $\psi$-Whittaker functional on the $\psi$-Kirillov model is given by evaluation at 1 .

To prove that $K_{2 l}$ acts on $\phi_{1}$ via $\eta$, it is enough to prove, as in [Casselman 1973], that $B(\mathcal{O})$ acts on $\phi_{1}$ via $\eta$ and that $N(\mathcal{O})$ fixes $\tilde{\pi}\left(\begin{array}{rr}0 & 1 \\ -\varpi^{2 l} & 0\end{array}\right) \phi_{1}$. The former is easy to verify using the definition of $\phi_{1}$. To address the latter, note that

$$
\tilde{\pi}\left(\begin{array}{cc}
0 & 1 \\
-\varpi^{2 l} & 0
\end{array}\right) \phi_{1}=\tilde{\pi}\left(\left(\begin{array}{cc}
0 & 1 \\
-1 & 0
\end{array}\right)\left(\begin{array}{cc}
\varpi^{2 l} & 0 \\
0 & 1
\end{array}\right)\right) \phi_{1}=\tilde{\pi}(w) \tau,
$$

where $\tau=\tilde{\pi}\left(\begin{array}{cl}\varpi^{2 l} & 0 \\ 0 & 1\end{array}\right) \phi_{1}$. It follows that $\tau(x)=\phi_{1}\left(\varpi^{2 l} x\right)$. To show that $N(\mathscr{O})$ fixes $\tilde{\pi}(w) \tau$, it suffices to show that the support of the function $\tilde{\pi}(w) \tau$ is in 0 . For this we need some information on the action of the Weyl group element $w$ on functions in the Kirillov model. This is given in terms of the formal Mellin transform of Jacquet and Langlands [1970].

The formal Mellin transform of any function $\xi$ in the Kirillov model for $\tilde{\pi}$ is a formal power series in $t$ defined for every character $v$ of $0^{\times}$as

$$
\widehat{\xi}(v, t):=\sum_{n \in \mathbb{Z}} \xi_{n}(v) t^{n}:=\sum_{n \in \mathbb{Z}}\left(\int_{u \in \mathbb{O}^{\times}} \xi\left(\varpi^{n} u\right) v(u) d u\right) t^{n} .
$$


Here we normalize the Haar measure $d u$ so that $0^{\times}$has volume 1 . For every $v$ there is a formal series $c(v, t)$ such that

$$
\widehat{\widetilde{\pi}(w) \xi}(v, t)=c(v, t) \widehat{\xi}\left(\omega_{0}^{-1} v^{-1}, z_{0}^{-1} t^{-1}\right)
$$

where $\omega_{0}$ is the restriction of the central character $\omega_{\widetilde{\pi}}$ to $\mathcal{O}^{\times}$and $z_{0}=\omega_{\widetilde{\pi}}(\varpi)$ (see Proposition 2.10 of [Jacquet and Langlands 1970]). Since $\tilde{\pi}$ is supercuspidal, it follows from Equation 2.18.1, Proposition 2.23, and the proof of Theorem 2.18 of the same paper that $c(v, t)$ is a monomial in $t$ of the form

$$
c(v, t)=c_{0}(v) t^{n_{v}}, \quad n_{v}=-c\left(\tilde{\pi} \otimes v^{-1}\right) \leq-2 .
$$

Using the definitions of $\phi_{1}$ and $\tau$ and the orthogonality of characters we get

$$
\widehat{\tau}(\nu, t)= \begin{cases}0 & \text { if } v \neq 1 \text { on }\left(\mathbb{O}^{\times}\right)^{2}, \\ c_{1} t^{-2 l} & \text { if } v=11 \text { on }\left(\mathbb{O}^{\times}\right)^{2} .\end{cases}
$$

where $c_{1}=\operatorname{vol}\left(\left(0^{\times}\right)^{2}\right)$. Hence we get

$$
\widehat{\tilde{\pi}(w) \tau}(v, t)= \begin{cases}0 & \text { if } v \neq \omega_{0}^{-1} \text { on }\left(\mathbb{O}^{\times}\right)^{2}, \\ c_{2}(v) t^{n_{v}+2 l} & \text { if } v=\omega_{0}^{-1} \text { on }\left(\mathbb{O}^{\times}\right)^{2} .\end{cases}
$$

for some nonzero constant $c_{2}(v)$. Now $N(\mathbb{O})$ fixes $\tilde{\pi}(w) \tau$ if the function $\tilde{\pi}(w) \tau$ is supported on $O$ ( since the conductor of $\psi$ is 0 ), and the latter is true if we show that $n_{v}+2 l \geq 0$ for any character $v$ which is $\omega_{0}^{-1}$ on $\left(\mathbb{O}^{\times}\right)^{2}$. In other words, we need to show that $c\left(\tilde{\pi} \otimes v^{-1}\right) \leq c(\tilde{\pi})$. (In fact, minimality of $\tilde{\pi}$ then forces equality, which would imply that the function $\tilde{\pi}(w) \tau$ is supported on $\tilde{O}^{\times}$.)

To prove this inequality, using the local Langlands correspondence (see [Kudla 1994] for instance), we consider the Langlands parameter $\varphi=\varphi(\tilde{\pi})$ of $\tilde{\pi}$ which is a two-dimensional irreducible representation of the Weil group $W_{F}$ of $F$. Since the residue characteristic is not 2 , we get that $\varphi$ is induced from a Galois regular character $\chi$ of $E^{*}$ for the unramified quadratic extension $E / F$. If $c(\varphi)$ denotes the local Artin conductor of $\varphi$, then we have, using Proposition 4(b) of $\S 4.3$ in [Serre 1967], $c(\tilde{\pi})=c(\varphi)=2 c(\chi)$. Since $v$ is $\omega_{0}^{-1}$ on $\left(\mathbb{O}^{\times}\right)^{2}$, we have $c(v) \leq$ $c\left(\omega_{\tilde{\pi}}\right)$ unless $c\left(\omega_{\tilde{\pi}}\right)=0$ and $c(v)=1$. Suppose the former condition holds. The central character is the determinant of the Langlands parameter and hence we get $\omega_{\tilde{\pi}}=\operatorname{det}(\varphi(\tilde{\pi}))=\operatorname{det}\left(\operatorname{ind}_{W_{E}}^{W_{F}}(\chi)\right)=\left.\omega_{E / F} \chi\right|_{F^{*}}$. Since $E / F$ is unramified we have $2 c\left(v^{-1}\right) \leq 2 c\left(\omega_{\tilde{\pi}}\right)=2 c\left(\left.\chi\right|_{F^{*}}\right) \leq 2 c(\chi)=c(\tilde{\pi})$. For every character $\kappa$ of $F^{*}$ we have the inequality $c(\tilde{\pi} \otimes \kappa) \leq \max \{c(\tilde{\pi}), 2 c(\kappa)\}$ (see $\S 4$ of [Gross 1988]). Applying this to $\kappa=v^{-1}$ and using the preceding information on the conductor of $v^{-1}$, we get the required inequality $-n_{v}=c\left(\tilde{\pi} \otimes v^{-1}\right) \leq \max \left\{c(\tilde{\pi}), 2 c\left(v^{-1}\right)\right\}=c(\tilde{\pi})=2 l$. If, on the other hand, $c\left(\omega_{\tilde{\pi}}\right)=0$ and $c(v)=1$, then $c\left(\tilde{\pi} \otimes v^{-1}\right) \leq c(\tilde{\pi})$ follows easily. 
We now state the results for the supercuspidal $L$-packets of cardinality four. We omit the proofs since they are minor modifications of the corresponding statements for the unramified supercuspidal $L$-packets that we just dealt with.

Proposition 3.3.6 (Unramified supercuspidal $L$-packet of cardinality four). Let $\widetilde{\sigma}$ denote a very cuspidal representation of $Z \widetilde{K}$ of level $l=1$ such that $\operatorname{Res}_{K}(\widetilde{\sigma})=$ $\sigma=\sigma_{1} \oplus \sigma_{2}$. Let $\left\{\pi_{1}, \pi_{1}^{\prime}, \pi_{2}, \pi_{2}^{\prime}\right\}$ be the corresponding L-packet of $G$. Then $c\left(\pi_{1}\right)=c\left(\pi_{1}^{\prime}\right)=c\left(\pi_{2}\right)=c\left(\pi_{2}^{\prime}\right)=2$. Moreover,

(i) Let $\eta$ be any character such that $\eta(-1)=\omega_{\sigma}(-1)$. If $\pi$ denotes any representation in the L-packet, then $\pi_{\eta}^{K_{1}}=\pi_{\eta}^{K_{1}^{\prime}}=(0)$.

(ii) Let $\eta$ be any character such that $\eta(-1)=\omega_{\sigma}(-1)$ and $c(\eta) \leq 1$. Then for all $m \geq 2$ we have

(a) $\operatorname{dim}\left(\pi_{1}\right){ }_{\eta}^{K_{m}^{\prime}}=\operatorname{dim}\left(\pi_{2}\right)_{\eta}^{K_{m}^{\prime}}=\operatorname{dim}\left(\pi_{1}^{\prime}\right)_{\eta}^{K_{m}}=\operatorname{dim}\left(\pi_{2}^{\prime}\right)_{\eta}^{K_{m}}=\left\lceil\frac{m-1}{2}\right\rceil$.

(b) $\operatorname{dim}\left(\pi_{1}\right)_{\eta}^{K_{m}}=\operatorname{dim}\left(\pi_{1}^{\prime}\right)_{\eta}^{K_{m}^{\prime}}=\operatorname{dim}\left(\pi_{2}\right)_{\eta}^{K_{m}}=\operatorname{dim}\left(\pi_{2}^{\prime}\right)_{\eta}^{K_{m}^{\prime}}=\left\lfloor\frac{m-1}{2}\right\rfloor$.

Corollary 3.3.7 (Test vectors for unramified supercuspidal $L$-packets of cardinality four). With notation as above let $\left\{\pi_{1}, \pi_{1}^{\prime}, \pi_{2}, \pi_{2}^{\prime}\right\}$ be the unramified supercuspidal L-packet of cardinality four. Let $\bar{\psi}$ be the character of $\mathbb{F}_{q}$ induced by $\psi$ by identifying $\mathbb{F}_{q}$ with $\mathscr{P}^{-1} / \mathcal{O}$. Without loss of generality assume that $\sigma_{1}$ is $\bar{\psi}$-generic. Then

(i) $\pi_{1}^{\prime}$ is $\psi$-generic, $\pi_{1}$ is $\psi_{\varpi}$-generic, $\pi_{2}^{\prime}$ is $\psi_{\epsilon}$-generic, and $\pi_{2}$ is $\psi_{\epsilon \varpi}$-generic.

(ii) Assume that $\tilde{\pi}$ is realized in its $\psi$-Kirillov model. The function $\phi_{1}$ of Proposition 3.3.5 is a newform for $\pi_{1}^{\prime}$. This further implies that $\tilde{\pi}(\alpha)\left(\phi_{1}\right)$ is a newform for $\pi_{1}, \tilde{\pi}(\gamma)\left(\phi_{1}\right)$ is a newform for $\pi_{2}^{\prime}$ and $\tilde{\pi}(\alpha \gamma)\left(\phi_{1}\right)$ is a newform for $\pi_{2}$. Finally, each of these newforms is a test vector for an appropriate Whittaker functional coming from (i).

We now consider the ramified supercuspidal $L$-packets. Let $\widetilde{\sigma}$ be a very cuspidal representation of $N_{\widetilde{G}}(\widetilde{I})$ of level $l \geq 1$. Let $\operatorname{Res}_{I} \widetilde{\sigma}=\sigma_{1} \oplus \sigma_{2}$ and let $\pi_{i}=\operatorname{ind}_{I}^{G}\left(\sigma_{i}\right)$. We call $\left\{\pi_{1}, \pi_{2}\right\}$ a ramified supercuspidal L-packet of $G$.

Proposition 3.3.8 (Ramified supercuspidal $L$-packets). Let $\left\{\pi_{1}, \pi_{2}\right\}$ be a ramified supercuspidal L-packet as above. Then $c\left(\pi_{1}\right)=c\left(\pi_{2}\right)=2 l+1$. We have

(i) For any character $\eta$ of $F^{*}$ such that $\eta(-1)=\omega_{\sigma}(-1)$ we have $\left(\pi_{1}\right)_{\eta}^{K_{2 l}}=$ $\left(\pi_{2}\right)_{\eta}^{K_{2 l}}=\left(\pi_{1}\right)_{\eta}^{K_{2 l}^{\prime}}=\left(\pi_{2}\right)_{\eta}^{K_{2 l}^{\prime}}=(0)$.

(ii) Let $\eta(-1)=\omega_{\sigma}(-1)$ and $c(\eta) \leq l$. For all $m \geq 2 l+1$ we have $\operatorname{dim}\left(\pi_{1}\right)_{\eta}^{K_{m}}=$ $\operatorname{dim}\left(\pi_{2}\right)_{\eta}^{K_{m}}=\operatorname{dim}\left(\pi_{1}\right)_{\eta}^{K_{m}^{\prime}}=\operatorname{dim}\left(\pi_{2}\right)_{\eta}^{K_{m}^{\prime}}=m-2 l$.

Proof. Since $\gamma$ conjugates $\pi_{1}$ to $\pi_{2}, \operatorname{dim}\left(\pi_{1}\right)_{\eta}^{K_{m}}=\operatorname{dim}\left(\pi_{2}\right)_{\eta}^{K_{m}}$ for all $m$. Furthermore, conjugation by $\alpha$ stabilizes both $\pi_{1}$ and $\pi_{2}$, which implies this same equality for $K_{m}^{\prime}$. 
That $\left(\pi_{1}\right)_{\eta}^{K_{2 l}}=(0)$ can be seen using Mackey theory [Kutzko 1977] as in the proof of Proposition 3.3.4 by considering the set of representatives for

$$
K_{2 l} \backslash \widetilde{G} / N_{\widetilde{G}}(\widetilde{I})
$$

given by

$\left\{\bar{n}(s) h(u) a_{r}: s \in \mathscr{P} / \mathscr{P}^{2 l}, u \in \mathbb{O}^{\times}, r \geq 0\right\} \cup\left\{\bar{n}(t) w h(u) a_{r}: t \in \mathscr{O} / \mathscr{P}^{2 l}, u \in \mathbb{O}^{\times}, r \geq 0\right\}$.

To prove (ii), we use induction on the level $m$ of the congruence subgroups $K_{m}$ or $K_{m}^{\prime}$. To begin the induction, we show that for $m=2 l+1$, all the relevant spaces are one-dimensional. It suffices, using Lemma 3.1.3, to prove that $\operatorname{dim}\left(\pi_{1}\right)_{\eta}^{K_{2 l+1}} \geq 1$. This is done as in the proof of Proposition 3.3.4. Note that $\widetilde{\sigma}_{\eta}^{U}$ is at least twodimensional and contains the span of $w_{1}$ and $w_{2}$. Since $\gamma$ conjugates $\sigma_{1}$ to $\sigma_{2}$, we get that both $\left(\sigma_{i}\right)_{\eta}^{U}$ are nonzero. Let us say that $w_{i} \in \sigma_{i}^{U}$. Then the corresponding $f_{w_{i}}$ is in $\left(\pi_{i}\right)_{\eta}^{K_{2 l+1}}$. To proceed with the induction argument, we note that, by Lemma 3.1.3, we need only show that for each $m \geq 2 l+2$, the dimension of $\left(\pi_{1}\right)_{\eta}^{K_{m}}$ is at least $m-2 l$. This follows from $\left(\pi_{1}\right)_{\eta}^{K_{m-1}} \oplus \tilde{\pi}(\beta)^{m-2 l-1}\left(\pi_{1}\right)_{\eta}^{K_{2 l+1}} \subset\left(\pi_{1}\right)_{\eta}^{K_{m}}$. This inclusion and the fact that the sum is direct is proved exactly as in the proof of Proposition 3.3.4.

Proposition 3.3.9 (Test vectors for ramified supercuspidal $L$-packets). Let $\left\{\pi_{1}, \pi_{2}\right\}$ be a ramified supercuspidal L-packet coming from a very cuspidal representation $\tilde{\sigma}$ of $N_{\widetilde{G}}(\widetilde{I})$ of level $l \geq 1$. One and only one of the $\pi_{i}$ is $\psi$-generic, say $\pi_{1}$. Then $\pi_{2}$ is $\psi_{\epsilon}$-generic. Let $\eta=\omega_{\sigma}$. Let $\phi_{1}$ and $\phi_{\epsilon}$ be as in Proposition 3.3.5. We have

(i) $\left(\pi_{1}\right)_{\eta}^{K_{2 l+1}}=\mathbb{C} \phi_{1}$ and $\left(\pi_{2}\right)_{\eta}^{K_{2 l+1}}=\mathbb{C} \phi_{\epsilon}$.

(ii) Any $\psi$-Whittaker functional is nonzero on $\phi_{1}$ and similarly any $\psi_{\epsilon}$-Whittaker functional is nonzero on $\phi_{\epsilon}$.

Proof. The proof is entirely analogous to the proof of Proposition 3.3.5. In fact, using the notation in that proof, it suffices now to show that the support of $\tilde{\pi}(w) \tau$ is in 0 , where $\tau=\tilde{\pi}\left(\alpha^{2 l+1}\right) \phi_{1}$. We can see as before that

$$
\widehat{\tilde{\pi}(w) \tau}(v, t)= \begin{cases}0 & \text { if } v \neq \omega_{0}^{-1} \text { on }\left(\mathcal{O}^{\times}\right)^{2}, \\ c_{1}(v) t^{n_{v}+2 l+1} & \text { if } v=\omega_{0}^{-1} \text { on }\left(\mathcal{O}^{\times}\right)^{2}\end{cases}
$$

where $\omega_{0}=\left.\omega_{\tilde{\pi}}\right|_{\mathcal{O}^{x}}$. As before, we need to show that if $v=\omega_{0}^{-1}$ on $\left(\mathbb{O}^{\times}\right)^{2}$, then in fact $n_{v}+2 l+1=0$. Since $\tilde{\pi}$ is a ramified supercuspidal representation, its Langlands parameter $\varphi=\varphi(\tilde{\pi})$ is a two dimensional irreducible representation of the Weil group $W_{F}$ of $F$ that is induced from a Galois regular character $\chi$ of $E^{*}$ for a ramified quadratic extension $E / F$. From Proposition $4(b)$ of $\S 4.3$ in [Serre 1967], we get that $c(\tilde{\pi})=c(\varphi)=c(\chi)+1$. By Theorem 3.3.3, $c(\tilde{\pi})=2 l+1$ which implies that $c(\chi)=2 l \geq 2$. This together with the fact that $E / F$ is ramified gives 
$2 c\left(\left.\chi\right|_{F^{*}}\right) \leq c(\chi)$. As in Proposition 3.3.5 we have $\omega_{\tilde{\pi}}=\left.\omega_{E / F} \chi\right|_{F^{*}}$. Hence we get $2 c\left(v^{-1}\right) \leq 2 c\left(\omega_{\tilde{\pi}}\right) \leq \max \left\{2,2 c\left(\left.\chi\right|_{F^{*}}\right)\right\} \leq c(\chi)<c(\chi)+1=c(\tilde{\pi})$. We deduce (using for instance $\S 4$ of [Gross 1988]) that $-n_{v}=c\left(\tilde{\pi} \otimes v^{-1}\right)=c(\tilde{\pi})=2 l+1$.

3.4. Comparison of conductor with other invariants. We begin by recording the following theorem relating the conductor of a representation $\pi$ of $G$ to the conductor of a minimal representation of $\widetilde{G}$ that determines the $L$-packet containing $\pi$.

Theorem 3.4.1 (Relation between $c(\pi)$ and $c(\tilde{\pi})$ ). Let $\pi$ be an irreducible admissible representation of $G=\mathrm{SL}_{2}(F)$. Let $\tilde{\pi}$ be a representation of $\widetilde{G}=\mathrm{GL}_{2}(F)$ whose restriction to $G$ contains $\pi$. Assume that $\tilde{\pi}$ is minimal, i.e., $c(\tilde{\pi} \otimes \chi) \geq c(\tilde{\pi})$ for all characters $\chi$ of $F^{*}$. Then $c(\pi)=c(\tilde{\pi})$.

Proof. If $\pi$ is a subquotient of a principal series representation $\pi(\chi)$ then the theorem follows from Propositions 3.2.4, 3.2.6, 3.2.8, 3.2.10 and 3.2.12 together with the easily verifiable fact that $\tilde{\pi}$ may be taken as $\operatorname{Ind}_{\widetilde{B}} \widetilde{G}(\chi \otimes \mathbb{1})$. If $\pi$ is a supercuspidal representation then the theorem follows from Propositions 3.3.4, 3.3.6 and 3.3.8 while keeping in mind that Kutzko's construction (Theorem 3.3.3) actually gives minimal supercuspidal representations $\tilde{\pi}$ of $\widetilde{G}$.

Now we relate the conductor of a representation $\pi$ of $G$ with the depth $\rho(\pi)$ of $\pi$ (a notion due to A. Moy and G. Prasad [1994]). We urge the reader to compare this theorem with a result from [Lansky and Raghuram 2003] where we determine such a relation for all discrete series representations of $D^{*}, \mathrm{GL}_{n}(F)$ and $\mathrm{GL}_{2}(D)$ for a central division algebra $D$ over $F$. We also mention in passing that considering the action of $\mathrm{GL}_{n}(F)$ on the Bruhat-Tits building of $\mathrm{SL}_{n}(F)$ we get that the depth of every representation in an $L$-packet of $\mathrm{SL}_{n}(F)$ is the same.

Theorem 3.4.2 (Relation between the conductor $c(\pi)$ and the depth $\rho(\pi)$ for $G$ ). Let $\pi$ be an irreducible representation of $G$. Let $\rho(\pi)$ be the depth of $\pi$.

(i) If $\pi$ is a subquotient of a principal series $\pi(\chi)$, then $\rho(\pi)=\max \{c(\pi)-1,0\}$.

(ii) If $\pi$ is an irreducible supercuspidal representation, then

$$
\rho(\pi)=\max \left\{\frac{c(\pi)-2}{2}, 0\right\} .
$$

Proof. The first statement is proved by the equalities $\rho(\pi)=\rho(\pi(\chi))=\rho(\chi)=$ $\max \{c(\chi)-1,0\}=\max \{c(\pi)-1,0\}$. He the first and second equality follow from [Moy and Prasad 1996] and the third from [Lansky and Raghuram 2003]. We omit the details of the proof of the second statement which can be proved almost exactly as in $\S 4$ of the latter paper. 


\section{Towards multiplicity one for newforms}

Given an irreducible representation $\pi$ of $G=\mathrm{SL}_{2}(F)$ and a character $\eta$ of $F^{*}$ such that $c_{\eta}(\pi)=c(\pi)$, one might ask if we have $\operatorname{dim} V_{\eta}^{K_{c(\pi)}}=1$. The answer is that this is often the case but not true in general. In fact we have $\operatorname{dim} V_{\eta}^{K_{c(\pi)}}=1$ unless $\pi$ is a representation in what we have called an unramified supercuspidal $L$-packet of cardinality two or if $\pi$ is an irreducible principal series representations $\pi(\chi)$ such that $\chi$ is not quadratic but $\left.\chi\right|_{0^{*}}$ is quadratic. For representations in these packets we get $\operatorname{dim} V_{\eta}^{K_{c(\pi)}}=2$.

Nevertheless, in all cases we have proved that an appropriate Whittaker functional is nonvanishing on some newform. This can be used to formulate a kind of a multiplicity one result if we consider the quotient of the space $V_{\eta}^{K_{c(\pi)}}$ of newforms by the kernel of this Whittaker functional. More precisely, if $\eta$ is such that $c_{\eta}(\pi)=c(\pi), \Psi$ is a nontrivial additive character of $F$ of conductor either 0 or $\mathscr{P}^{-1}$ such that $\pi$ is $\Psi$-generic, and $\Lambda_{\Psi}$ is a $\Psi$-Whittaker functional for $\pi$, then we have

$$
\operatorname{dim} \frac{V_{\eta}^{K_{c(\pi)}}}{V_{\eta}^{K_{c(\pi)}} \cap \operatorname{kernel}\left(\Lambda_{\Psi}\right)}=1 .
$$

Another possibility is to consider some canonical nondegenerate bilinear form on the space $V^{K_{c(\pi)}}$ and consider the orthogonal complement of the subspace

$$
V_{\eta}^{K_{c(\pi)}} \cap \operatorname{kernel}\left(\Lambda_{\Psi}\right)
$$

as a candidate for a one-dimensional space of newforms. Then the multiplicity one result is formulated as $\operatorname{dim}\left(V_{\eta}^{K_{c(\pi)}} \cap \operatorname{kernel}\left(\Lambda_{\Psi}\right)\right)^{\perp}=1$.

\section{Acknowledgments}

We thank Benedict Gross, Dipendra Prasad, Brooks Roberts and Paul Sally Jr. for some helpful correspondence.

\section{References}

[Bump 1997] D. Bump, Automorphic forms and representations, Cambridge Studies in Advanced Mathematics 55, Cambridge University Press, Cambridge, 1997. MR 97k:11080 Zbl 0868.11022 [Casselman 1973] W. Casselman, "On some results of Atkin and Lehner", Math. Ann. 201 (1973), 301-314. MR 49 \#2558 Zbl 0239.10015

[Deligne 1973] P. Deligne, "Formes modulaires et représentations de GL(2)", pp. 55-105 in Modular functions of one variable, II (Antwerp, 1972), edited by P. Deligne and W. Kuyk, Lecture Notes in Math 349, Springer, Berlin, 1973. MR 50 \#240 Zbl 0271.10032

[Gelbart and Knapp 1982] S. S. Gelbart and A. W. Knapp, " $L$-indistinguishability and $R$ groups for the special linear group", Adv. in Math. 43:2 (1982), 101-121. MR 83j:22009 Zbl 0493.22005 
[Gel'fand et al. 1969] I. M. Gel'fand, M. I. Graev, and I. I. Pyatetskii-Shapiro, Generalized functions, VI: Representation theory and automorphic functions, Academic Press, 1969. MR 91g:11052 Zbl 0235.43012

[Gross 1988] B. H. Gross, "Local orders, root numbers, and modular curves", Amer. J. Math. 110:6 (1988), 1153-1182. MR 90b:11053 Zbl 0675.12011

[Gross and Prasad 1991] B. H. Gross and D. Prasad, "Test vectors for linear forms", Math. Ann. 291:2 (1991), 343-355. MR 92k:22028 Zbl 0768.22004

[Jacquet and Langlands 1970] H. Jacquet and R. P. Langlands, Automorphic forms on GL(2), Lecture Notes in Mathematics 114, Springer, Berlin, 1970. MR 53 \#5481 Zbl 0236.12010

[Jacquet et al. 1981] H. Jacquet, I. I. Piatetski-Shapiro, and J. Shalika, "Conducteur des représentations du groupe linéaire”, Math. Ann. 256:2 (1981), 199-214. MR 83c:22025 Zbl 0443.22013

[Kudla 1994] S. S. Kudla, "The local Langlands correspondence: the non-Archimedean case", pp. 365-391 in Motives (Seattle, WA, 1991), vol. 2, edited by U. Jannsen et al., Proc. Sympos. Pure Math. 55, Amer. Math. Soc., Providence, RI, 1994. MR 95d:11065 Zbl 0811.11072

[Kutzko 1977] P. C. Kutzko, "Mackey's theorem for nonunitary representations", Proc. Amer. Math. Soc. 64:1 (1977), 173-175. MR 56 \#533 Zbl 0375.22005

[Kutzko 1978a] P. C. Kutzko, "On the supercuspidal representations of Gl2", Amer. J. Math. 100:1 (1978), 43-60. MR 58 \#22411a Zbl 0417.22012

[Kutzko 1978b] P. C. Kutzko, "On the supercuspidal representations of Gl2. II", Amer. J. Math. 100:4 (1978), 705-716. MR 58 \#22411b Zbl 0421.22012

[Kutzko and Sally 1983] P. C. Kutzko and P. J. Sally, Jr., "All supercuspidal representations of $\mathrm{SL}_{l}$ over a $p$-adic field are induced", pp. 185-196 in Representation theory of reductive groups (Park City, UT, 1982), edited by P. C. Trombi, Progr. Math. 40, Birkhäuser, Boston, 1983. MR 85i:22029 Zbl 0538.22011

[Labesse and Langlands 1979] J.-P. Labesse and R. P. Langlands, " $L$-indistinguishability for SL(2)”, Canad. J. Math. 31:4 (1979), 726-785. MR 81b:22017 Zbl 0421.12014

[Lansky and Raghuram 2003] J. Lansky and A. Raghuram, "On the correspondence of representations between GL( $n)$ and division algebras", Proc. Amer. Math. Soc. 131:5 (2003), 1641-1648. MR 2003m:22021 Zbl 1027.22017

[Lansky and Raghuram 2004] J. Lansky and A. Raghuram, "Conductors and newforms for $U(1,1)$ ", Proc. Indian Acad. Sci. Math. Sci. 114:4 (2004), 319-343. MR 2005k:11099 Zbl 1059.11039

[Manderscheid 1984] D. Manderscheid, "On the supercuspidal representations of SL $\mathrm{SL}_{2}$ and its twofold cover”, Math. Ann. 266:3 (1984), 287-295, 297-305. MR 85d:22032 Zbl 0507.22014

[Mann 2001] W. R. Mann, Local level raising for GL(n), Ph.D. thesis, Harvard University, Cambridge, MA, 2001.

[Moy and Prasad 1994] A. Moy and G. Prasad, "Unrefined minimal $K$-types for $p$-adic groups", Invent. Math. 116:1-3 (1994), 393-408. MR 95f:22023 Zbl 0804.22008

[Moy and Prasad 1996] A. Moy and G. Prasad, "Jacquet functors and unrefined minimal $K$-types", Comment. Math. Helv. 71:1 (1996), 98-121. MR 97c:22021 Zbl 0860.22006

[Prasad and Raghuram 2000] D. Prasad and A. Raghuram, "Kirillov theory for $\mathrm{GL}_{2}(\mathscr{D})$ where $\mathscr{D}$ is a division algebra over a non-Archimedean local field", Duke Math. J. 104:1 (2000), 19-44. MR 2001i:22024 Zbl 0958.22010

[Schmidt 2002] R. Schmidt, "Some remarks on local newforms for GL(2)", J. Ramanujan Math. Soc. 17:2 (2002), 115-147. MR 2003g:11056 Zbl 0997.11040 
[Serre 1967] J.-P. Serre, "Local class field theory", pp. 128-161 in Algebraic number theory: Proceedings of the instructional conference (Brighton, Sussex, 1965), Thompson, Washington, DC, 1967. Reprinted Academic Press, London, 1986. MR 36 \#3753 Zbl 0959.13010

[Shelstad 1979] D. Shelstad, "Notes on L-indistinguishability", pp. 193-203 in Automorphic forms, representations and L-functions (Corvallis, OR., 1977), vol. 2, edited by A. Borel and W. Casselman, Proc. Sympos. Pure Math. 33, Amer. Math. Soc., Providence, 1979. Based on a lecture of R. P. Langlands. MR 81m:12018 Zbl 0421.12015

[Shimizu 1977] H. Shimizu, "Some examples of new forms", J. Fac. Sci. Univ. Tokyo Sect. IA Math. 24:1 (1977), 97-113. MR 56 \#5436 Zbl 0359.10023

[Waldspurger 1985] J.-L. Waldspurger, "Quelques propriétés arithmétiques de certaines formes automorphes sur GL(2)”, Compositio Math. 54:2 (1985), 121-171. MR 87g:11061a Zbl 0567.10022

Received August 30, 2005.

JOSHUA M. LANSKY

DePartment of Mathematics And Statistics

AMERICAN UNIVERSITY

4400 Massachusetts Avenue, NW

WASHINGTON, DC 20016

UNITED STATES

lansky@american.edu

http://www.american.edu/faculty/lansky/

\author{
A. RAGHURAM \\ DEPARTMENT OF MATHEMATICS \\ OKLAHOMA STATE UNIVERSITY \\ 401 Mathematical SCIENCES \\ STILLWATER, OK 74078 \\ UNITED STATES \\ araghur@math.okstate.edu \\ http://www.math.okstate.edu/ raghuram/
}

